\title{
A multiscale overlapped coupling formulation for large-deformation strain localization
}

\author{
WaiChing Sun • Alejandro Mota
}

Received: 18 September 2013 / Accepted: 7 April 2014 / Published online: 3 May 2014

(C) Springer-Verlag Berlin Heidelberg 2014

\begin{abstract}
We generalize the multiscale overlapped domain framework to couple multiple rate-independent standard dissipative material models in the finite deformation regime across different length scales. We show that a fully coupled multiscale incremental boundary-value problem can be recast as the stationary point that optimizes the partitioned incremental work of a three-field energy functional. We also establish inf-sup tests to examine the numerical stability issues that arise from enforcing weak compatibility in the three-field formulation. We also devise a new block solver for the domain coupling problem and demonstrate the performance of the formulation with one-dimensional numerical examples. These simulations indicate that it is sufficient to introduce a localization limiter in a confined region of interest to regularize the partial differential equation if loss of ellipticity occurs.
\end{abstract}

Keywords Domain coupling - Variational principle . Energy based coupling method $\cdot$ Multiscale modeling

\section{Introduction}

When subjected to certain loading conditions many materials, such as metals and soils, develop concentrated strain in a narrow zone as opposed to homogeneous deformation. Since

W. Sun $(\bowtie)$

Department of Civil Engineering and Engineering Mechanics, Columbia University in the City of New York, New York, NY 10027, USA

e-mail:wsun@columbia.edu

A. Mota

Mechanics of Materials Department, Sandia National Laboratories, Livermore, CA 94550, USA strain localization may lead to the eventual failure of materials, this phenomenon is of significant importance to modern engineering applications.

The objective of this work is to introduce concurrent coupling between sub-scale and macro-scale simulations for inelastic materials that are prone to strain localization. Since it is not feasible to conduct sub-scale simulations on macroscopic problems, we use the domain coupling method such that computational resources can be efficiently allocated to regions of interest $[14,23,24,30]$. To the best of our knowledge, this is the first work focusing on utilizing the domain coupling method to model strain localization in inelastic materials undergoing large deformation.

Nevertheless, modeling strain localization with the conventional finite element method may lead to spurious meshdependent results due to the loss of ellipticity at the onset of strain localization [31]. To circumvent the loss of material stability, a localization limiter must be introduced. This localization limiter can be (i) a nonlocal or gradient model in which the constitutive response is governed by the gradient or the integral of at least one local internal variable [8], (ii) a rate dependent constitutive law [10], (iii) a multiphysics model that provides regularization through coupling with heat or pore-fluid diffusion $[1,27,33,34,39]$ or (iv) a formulation that allows displacement discontinuities $[9,15,16,28,38]$. These methods may help to maintain the uniqueness of the governing equations and eliminate the pathological dependence of the numerical solutions on the mesh size. Herein, we use a variational nonlocal regularization scheme to deal with material instabilities [29].

\subsection{Previous work}

Over the last two decades, an extensive body of work has been proposed to simulate strain localization phenomena in 
different materials. Strain localization often occurs at a scale several orders of magnitude smaller than the macroscopic scale at which classical continuum mechanics applies, therefore it is difficult to account properly for the inherent fine scale of the mechanical behavior to ensure correctness.

As pointed out by Aubertin et al. [2], methods used to incorporate fine scale mechanical properties into macroscopic analysis can be classified into three categories. The first method is to model the inherent multiscale character and introduce a length scale through phenomenological laws. For instance, Fleck and Hutchinson [22] introduce a strain gradient into constitutive models such that multiple material length parameters can be defined for the field equations corresponding to different dominant mechanisms. For problems where the deformation band thickness is much smaller than the mesh size, enhancement basis functions or localization elements can be inserted to idealize the deformation band with embedded strongly or weakly discontinuous displacement fields $[15,16,38]$.

Another class of methods is to incorporate microstructural information from unit cells to compute effective macroscopic properties on the coarse domain [11,21]. By passing stress, internal variables and dislocation information, this approach provides a cohesive law to the coarse domain and introduces a length scale that regularizes the governing equation. Kouznetsova et al. [26] introduce a gradientenhanced homogenization scheme in which the macroscopic stress, strain measure and their gradients are obtained from solutions of boundary value problems applied on representative volume elements. A similar unit cell approach has been applied to porous flow problems recently by White et al. [37] and Sun et al. [32] to conduct large scale flow simulations on tomographic images. In both cases, the quality of the hierarchical multiscale analysis depends on the existence of a representative elementary volume and the design of sequential coupling schemes that establish an information exchange across scales.

The third approach relies on the identification of a region or sub-domain that is of high interest, then computational efforts are concentrated in this most critical region where strain localization occurs. The fine-scale processes in this region are represented by using appropriate constitutive laws, special numerical methods or fine meshes. This region is thus connected to a coarse region in which a simpler low-cost macroscopic model is used. The advantage of this method is the ability to effectively allocate computational resources to sub-domains based on the need to characterize their fine-scale behavior. Previous work has established a variety of methods that successfully couple discrete and continuum models (e.g. Bauman et al. [6,30], Prudhomme et al. [7]), classical local and nonlocal elastic continuum (e.g. Han and Lubineau [24]) and structural elements with various mesh refinements (e.g. Ben Dhia and Rateau [14]).
Nevertheless, to the best of our knowledge, the domain coupling method has not yet been applied to model strain localization in materials exhibiting history dependent mechanical behavior such as plasticity or damage.

\subsection{Proposed method}

We investigate how to use the domain coupling method to conduct numerical simulations that are capable of capturing strain localization without compromising computational efficiency, numerical stability or accuracy. First introduced by Ben Dhia [12], the domain coupling method is designed to provide a flexible way to superpose and couple multiple models in a globally defined domain such that computational resources can be allocated to the most critical region of interest. The key components of the domain coupling method include a coarse and a fine domain which are often modeled by different constitutive laws or meshes or both, an overlapped domain in which these two sub-domains are coupled through a partition of unity, and a Lagrange multiplier to enforce the compatibility between them. Previously, domain coupling methods were used for coupling atomicto-continuum field equations, as discussed in Bauman et al. [6]. Guidault and Belytschko [23] adopt the framework to a continuum-to-continuum coupling problem and find that this formulation is well-posed if and only if a proper combination of weight functions and compatibility enforcer is used. Bauman et al. [6] derive an inf-sup condition by introducing a normed product space for coarse and fine displacements. This inf-sup condition is used to assess stability of the coupled atomic-to-continuum method. Han and Lubineau [24] extend this work to couple local elastic continua to nonlocal elastic continua in the small strain regime. In all these works, the materials of interest are all path-independent and therefore require no internal variables.

In this work, the point of departure is the extension of the domain coupling framework to link a potentially nonlocallyregularized model to a local standard dissipative model via an incremental variational principle in finite strains. By applying a partition of unity to the Helmholtz free energy density and the external potential, we derive a variational statement for the concurrently coupled models in each of the partitioned sub-domains. Various methods used to enforce compatibility between the coarse and fine deformation mappings are analyzed. One-dimensional numerical examples are provided to demonstrate the computational efficiency, flexibility and accuracy of the proposed approach.

\section{Finite element formulation}

In this section, we derive a three-field finite-deformation finite element formulation of the overlapped domain prob- 


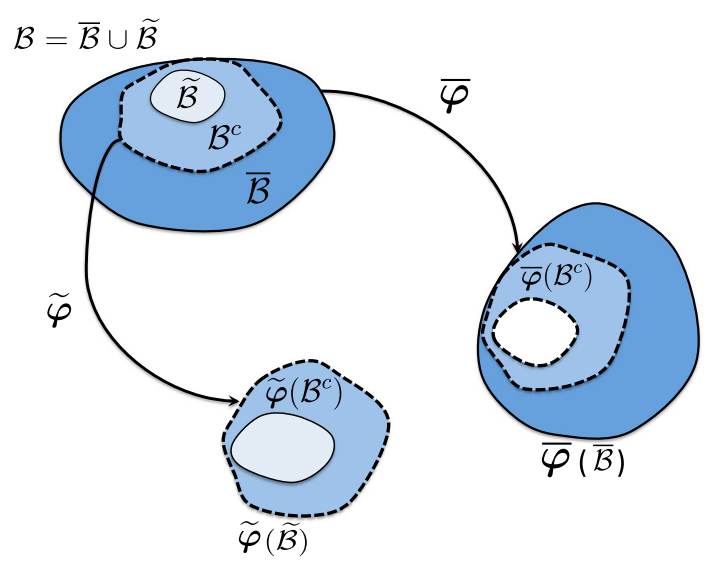

Fig. 1 Sub-domains $\overline{\mathcal{B}}$ and $\widetilde{\mathcal{B}}$ of the dissipative solid body $\mathcal{B}$ subjected to deformation $\bar{\varphi}$ and $\widetilde{\varphi}$

lem by applying a partition of unity to the incremental energy density functions and enforcing weak compatibility between the deformation mappings of the coarse and fine scales.

\subsection{Domain partition}

Consider a body $\mathcal{B}$ partitioned into two sub-domains $\overline{\mathcal{B}}$ and $\widetilde{\mathcal{B}}$ such that $\mathcal{B}=\overline{\mathcal{B}} \cup \widetilde{\mathcal{B}}$ and an overlapped region $\mathcal{B}^{c}=$ $\overline{\mathcal{B}} \cap \widetilde{\mathcal{B}}$, as shown in Fig. 1. The coarse domain $\overline{\mathcal{B}}$ occupies a usually larger portion of the body despite being associated with fewer degrees of freedom per volume. Conversely, the fine domain $\widetilde{\mathcal{B}}$ occupies a usually smaller portion of the body where critical mechanisms may occur and is hence modeled by a more sophisticated constitutive law or meshed with more degrees of freedom or both.

For consistency, henceforth denote as $\overline{(\cdot)}$ and $\widetilde{(\cdot)}$ quantities related to $\overline{\mathcal{B}}$ and $\widetilde{\mathcal{B}}$ respectively. The Dirichlet boundary is the union of the partitioned Dirichlet boundaries, i.e., $\partial_{\varphi} \mathcal{B}=\partial_{\bar{\varphi}} \overline{\mathcal{B}} \cup \partial_{\tilde{\varphi}} \widetilde{\mathcal{B}}$. The partitioned body may contain an overlapped Dirichlet boundary $\partial_{\varphi} \mathcal{B}^{c}$, i.e., $\partial_{\varphi} \mathcal{B}^{c}=\partial_{\bar{\varphi}} \overline{\mathcal{B}} \cap \partial_{\widetilde{\varphi}} \widetilde{\mathcal{B}}$. The von Neumann boundary is $\partial_{\boldsymbol{T}} \mathcal{B}=\partial_{\bar{T}} \overline{\mathcal{B}} \cup \partial_{\widetilde{T}} \widetilde{\mathcal{B}}$, with an overlapped von Neumann boundary $\partial_{T} \mathcal{B}^{c}=\partial_{\bar{T}} \overline{\mathcal{B}} \cap \partial_{\widetilde{T}} \widetilde{\mathcal{B}}$. Note that it is possible to partition the body either in the reference or the current configurations. Our domain coupling formulation, however, is derived with respect to the reference configuration. This treatment is suitable for hyper-elasticity or variational plasticity models used in conjunction with a total Lagrangian finite element formulation. On the other hand, for spatial material models a partition in the current configuration should be used.

\subsection{Domain coupling formulation}

The deformation of the continuum inside the coarse and fine sub-domains can be described by the mappings $\overline{\boldsymbol{x}}=$ $\overline{\boldsymbol{\varphi}}(\boldsymbol{X}, t): \overline{\mathcal{B}} \times\left[t_{1}, t_{2}\right] \rightarrow \mathbb{R}^{3}$ and $\widetilde{\boldsymbol{x}}=\widetilde{\boldsymbol{\varphi}}(\boldsymbol{X}, t): \widetilde{\mathcal{B}} \times\left[t_{1}, t_{2}\right] \rightarrow$
$\mathbb{R}^{3}$, with the coarse and fine deformation gradients defined by $\overline{\boldsymbol{F}}:=\operatorname{Grad} \overline{\boldsymbol{\varphi}}$ and $\widetilde{\boldsymbol{F}}:=\operatorname{Grad} \widetilde{\boldsymbol{\varphi}}$ respectively.

The boundary $\partial \overline{\mathcal{B}}$, with the unit normal $\bar{N}$, is the union of a displacement boundary $\partial_{\bar{\varphi}} \overline{\mathcal{B}}$, where the boundary displacement $\bar{\chi}: \partial_{\bar{\varphi}} \overline{\mathcal{B}} \times\left[t_{1}, t_{2}\right] \rightarrow \mathbb{R}^{3}$ is prescribed, and a traction boundary $\partial_{\bar{T}} \overline{\mathcal{B}}$, where the traction $\overline{\boldsymbol{T}}: \partial_{\bar{T}} \overline{\mathcal{B}} \times\left[t_{1}, t_{2}\right] \rightarrow \mathbb{R}^{3}$ is applied $\left(\partial_{\bar{\varphi}} \overline{\mathcal{B}} \cap \partial_{\bar{T}} \overline{\mathcal{B}}=\emptyset\right)$. Conversely, the boundary $\partial \widetilde{\mathcal{B}}$, with the unit normal $\widetilde{N}$, is the union of a displacement boundary $\partial_{\widetilde{\varphi}} \widetilde{\mathcal{B}}$, where the boundary displacement $\widetilde{\chi}: \partial_{\widetilde{\varphi}} \widetilde{\mathcal{B}} \times\left[t_{1}, t_{2}\right] \rightarrow$ $\mathbb{R}^{3}$ is prescribed, and a traction boundary $\partial_{\widetilde{T}} \widetilde{\mathcal{B}}$, where the traction $\widetilde{\boldsymbol{T}}: \partial_{\widetilde{T}} \widetilde{\mathcal{B}} \times\left[t_{1}, t_{2}\right] \rightarrow \mathbb{R}^{3}$ is applied $\left(\partial_{\widetilde{\varphi}} \widetilde{\mathcal{B}} \cap \partial_{\widetilde{T}} \widetilde{\mathcal{B}}=\emptyset\right)$. Let $\overline{\boldsymbol{B}}: \overline{\mathcal{B}} \times\left[t_{1}, t_{2}\right] \rightarrow \mathbb{R}^{3}$ and $\widetilde{\boldsymbol{B}}: \widetilde{\mathcal{B}} \times\left[t_{1}, t_{2}\right] \rightarrow \mathbb{R}^{3}$ be the coarse and fine body forces described in the reference configuration, respectively.

The point of departure for the domain coupling formulation is the introduction of a partition of unity to the Helmholtz free-energy density and external work density. Augmented with a constraint term to enforce compatibility, the total free energy becomes a three-field energy functional,

$$
\begin{aligned}
& \Phi[\overline{\boldsymbol{\varphi}}, \widetilde{\boldsymbol{\varphi}}, \boldsymbol{\phi}]:= \Phi^{\mathrm{int}}[\overline{\boldsymbol{\varphi}}, \widetilde{\boldsymbol{\varphi}}]-\Phi^{\mathrm{ext}}[\overline{\boldsymbol{\varphi}}, \widetilde{\boldsymbol{\varphi}}]+\Lambda[\overline{\boldsymbol{\varphi}}, \widetilde{\boldsymbol{\varphi}}, \boldsymbol{\phi}], \\
& \Phi^{\mathrm{int}}[\overline{\boldsymbol{\varphi}}, \widetilde{\boldsymbol{\varphi}}]:= \int_{\mathcal{B}}[\chi \bar{W}(\overline{\boldsymbol{F}}, \overline{\boldsymbol{Z}})+(1-\chi) \widetilde{W}(\widetilde{\boldsymbol{F}}, \widetilde{\boldsymbol{Z}})] d V, \\
& \Phi^{\mathrm{ext}}[\overline{\boldsymbol{\varphi}}, \widetilde{\boldsymbol{\varphi}}]:= \int_{\mathcal{B}}[\psi \overline{\boldsymbol{B}} \cdot \overline{\boldsymbol{\varphi}}+(1-\psi) \widetilde{\boldsymbol{B}} \cdot \widetilde{\boldsymbol{\varphi}}] d V \\
&+\int_{\partial_{T} \mathcal{B}}[\psi \overline{\boldsymbol{T}} \cdot \overline{\boldsymbol{\varphi}}+(1-\psi) \widetilde{\boldsymbol{T}} \cdot \widetilde{\boldsymbol{\varphi}}] d S, \\
& \Lambda[\overline{\boldsymbol{\varphi}}, \widetilde{\boldsymbol{\varphi}}, \boldsymbol{\phi}]:= \int_{\mathcal{B}^{c}}\left[\boldsymbol{\phi} \cdot(\overline{\boldsymbol{\varphi}}-\widetilde{\boldsymbol{\varphi}})+\kappa \ell^{2} \operatorname{Grad} \boldsymbol{\phi}:\right. \\
&(\operatorname{Grad} \overline{\boldsymbol{\varphi}}-\operatorname{Grad} \widetilde{\boldsymbol{\varphi}})] d V,
\end{aligned}
$$

where $\bar{W}(\overline{\boldsymbol{F}}, \overline{\boldsymbol{Z}})$ and $\widetilde{W}(\widetilde{\boldsymbol{F}}, \widetilde{\boldsymbol{Z}})$ are the Helmholtz freeenergy densities of the coarse and fine sub-domains and $\bar{Z}$ and $\widetilde{\boldsymbol{Z}}$ are corresponding collections of internal variables, $\chi: \mathcal{B} \rightarrow[0,1]$ and $\psi: \mathcal{B} \rightarrow[0,1]$ are weighting functions of the Helmholtz free-energy density and the external work density. Both weighting functions hold the following properties in the non-overlapped domain,

$\chi(\boldsymbol{X})=\psi(\boldsymbol{X})= \begin{cases}1, & \boldsymbol{X} \in \overline{\mathcal{B}} \backslash \mathcal{B}^{c} \\ 0, & \boldsymbol{X} \in \widetilde{\mathcal{B}} \backslash \mathcal{B}^{c} .\end{cases}$

In the overlapped domain, $\chi(\boldsymbol{X})$ and $\psi(\boldsymbol{X})$ may be chosen as constant or $C^{k}$ functions. As pointed out by Guidault and Belytschko [23] and Han and Lubineau [24], valid choices for the weighting functions depends on how the compatibility is enforced in the overlapped domain. The additional constraint term $\Lambda[\bar{\varphi}, \widetilde{\varphi}, \phi]$ is introduced to minimize the compatibility error between the coarse and fine domains, where $\kappa$ is a dimensionless parameter with $\kappa=0$ or $\kappa=1$ for $L_{2}$ or $H^{1}$ compatibility errors, respectively. The parameter $\ell$ is a 
length first introduced by Guidault and Belytschko [23] so that the constraint remains dimensionless.

\subsection{Variational statement}

Assume that $\overline{\boldsymbol{\varphi}} \in \bar{U}:=\left(W_{2}^{1}(\overline{\mathcal{B}})\right)^{3}, \widetilde{\boldsymbol{\varphi}} \in \widetilde{U}:=\left(W_{2}^{1}(\widetilde{\mathcal{B}})\right)^{3}$ and $\boldsymbol{\phi} \in V:=\left(W_{2}^{1}\left(\mathcal{B}^{c}\right)\right)^{3}$ in which $W_{2}^{1}(\mathcal{B})$ is the Sobolev space of square-integrable functions with square-integrable first derivatives. The functional (2.1) is optimized by applying variations with respect to the independent fields $\bar{\varphi}, \widetilde{\varphi}$ and $\boldsymbol{\phi}$. Define test functions corresponding to these fields as $\bar{\xi} \in \bar{U}, \widetilde{\xi} \in \widetilde{U}$ and $\eta \in V$, with $\bar{\xi}=\mathbf{0}$ on $\partial_{\bar{\varphi}} \overline{\mathcal{B}}$ and $\widetilde{\xi}=\mathbf{0}$ on $\partial_{\widetilde{\varphi}} \widetilde{\mathcal{B}}$. The variations follow as

$$
\begin{aligned}
D \Phi[\overline{\boldsymbol{\varphi}}, \widetilde{\boldsymbol{\varphi}}, \boldsymbol{\phi}](\overline{\boldsymbol{\xi}})= & \int_{\mathcal{B}}(\chi \overline{\boldsymbol{P}}: \operatorname{Grad} \overline{\boldsymbol{\xi}}-\psi \overline{\boldsymbol{B}} \cdot \overline{\boldsymbol{\xi}}) d V \\
& +\int_{\mathcal{B}^{c}}\left(\boldsymbol{\phi} \cdot \overline{\boldsymbol{\xi}}+\kappa \ell^{2} \operatorname{Grad} \boldsymbol{\phi}: \operatorname{Grad} \overline{\boldsymbol{\xi}}\right) d V \\
& -\int_{\partial_{T} \mathcal{B}} \psi \overline{\boldsymbol{T}} \cdot \bar{\xi} d S=0,
\end{aligned}
$$

$D \Phi[\overline{\boldsymbol{\varphi}}, \widetilde{\boldsymbol{\varphi}}, \boldsymbol{\phi}](\widetilde{\boldsymbol{\xi}})=\int_{\mathcal{B}}[(1-\chi) \widetilde{\boldsymbol{P}}: \operatorname{Grad} \widetilde{\boldsymbol{\xi}}-(1-\psi) \widetilde{\boldsymbol{B}} \cdot \widetilde{\boldsymbol{\xi}}] d V$

$$
-\int_{\mathcal{B}^{c}}\left(\boldsymbol{\phi} \cdot \widetilde{\xi}+\kappa \ell^{2} \operatorname{Grad} \boldsymbol{\phi}: \operatorname{Grad} \widetilde{\xi}\right) d V
$$$$
-\int_{\partial_{T} \mathcal{B}}(1-\psi) \widetilde{\boldsymbol{T}} \cdot \widetilde{\boldsymbol{\xi}} d S=0,
$$

$D \Phi[\overline{\boldsymbol{\varphi}}, \widetilde{\boldsymbol{\varphi}}, \boldsymbol{\phi}](\boldsymbol{\eta})=\int_{\mathcal{B}^{c}}\left[\eta \cdot(\overline{\boldsymbol{\varphi}}-\widetilde{\boldsymbol{\varphi}})+\kappa \ell^{2} \operatorname{Grad} \boldsymbol{\eta}:\right.$

$$
(\operatorname{Grad} \bar{\varphi}-\operatorname{Grad} \widetilde{\varphi})] d V=0 .
$$

Here we assume that the mechanical responses of both domains $\overline{\mathcal{B}}$ and $\widetilde{\mathcal{B}}$ can be adequately described by standard dissipative solid models such that $\overline{\boldsymbol{P}}=\partial \bar{W} / \partial \overline{\boldsymbol{F}}$ and $\widetilde{\boldsymbol{P}}=$ $\partial \widetilde{W} / \partial \widetilde{\boldsymbol{F}}$ which are the first Piola-Kirchhoff stresses corresponding to the coarse and fine sub-domains, respectively. The corresponding Euler-Lagrange equations for (2.1) in the non-overlapped domains are simply the balance of linear momentum equation corresponding to the coarse and fine mappings from their reference to the current configurations, i.e.,

$$
\begin{aligned}
\operatorname{Div} \overline{\boldsymbol{P}}+\overline{\boldsymbol{B}} & =\mathbf{0} \quad \text { in } \quad \overline{\mathcal{B}} \backslash \mathcal{B}^{c}, \\
\overline{\boldsymbol{P}} \boldsymbol{N} & =\overline{\boldsymbol{T}} \quad \text { on } \quad \partial_{\bar{T}} \overline{\mathcal{B}} \backslash \partial_{T} \mathcal{B}^{c},
\end{aligned}
$$

and

$$
\begin{aligned}
\operatorname{Div} \widetilde{\boldsymbol{P}}+\widetilde{\boldsymbol{B}} & =\mathbf{0} \quad \text { in } \widetilde{\mathcal{B}} \backslash \mathcal{B}^{c}, \\
\widetilde{\boldsymbol{P}} \boldsymbol{N} & =\widetilde{\boldsymbol{T}} \quad \text { on } \quad \partial_{\widetilde{T}} \widetilde{\mathcal{B}} \backslash \partial_{T} \mathcal{B}^{c} .
\end{aligned}
$$

In the overlapped domain, the Euler-Lagrange equations read,

$$
\begin{aligned}
& \operatorname{Div}(\chi \overline{\boldsymbol{P}})+\psi \overline{\boldsymbol{B}}-\boldsymbol{\phi}+\kappa \ell^{2} \operatorname{Div} \operatorname{Grad} \boldsymbol{\phi}=\mathbf{0} \text { in } \mathcal{B}^{c}, \\
& \operatorname{Div}[(1-\chi) \widetilde{\boldsymbol{P}}]+(1-\psi) \widetilde{\boldsymbol{B}}+\boldsymbol{\phi}-\kappa \ell^{2} \operatorname{Div} \operatorname{Grad} \boldsymbol{\phi}=\mathbf{0} \text { in } \mathcal{B}^{c} \text {, } \\
& \bar{\varphi}-\widetilde{\varphi}-\kappa \ell^{2} \operatorname{Div} \operatorname{Grad}(\bar{\varphi}-\widetilde{\varphi})=\mathbf{0} \text { in } \mathcal{B}^{c}, \\
& {[\chi \overline{\boldsymbol{P}}+(1-\chi) \widetilde{\boldsymbol{P}}] N=\psi \overline{\boldsymbol{T}}+(1-\psi) \widetilde{\boldsymbol{T}} \text { on }} \\
& \partial_{T} \mathcal{B}^{c} .
\end{aligned}
$$

\subsection{Discrete variational statement}

Next, we introduce the spatial discretization for the primary fields and test functions. In particular, let the Lagrange multiplier $\phi$ and coarse configuration mapping $\bar{\varphi}$ be spanned by the same set of interpolation functions. Note that it is possible to use three finite dimensional bases for the Lagrange multiplier and deformation mappings $\bar{\varphi}$ and $\widetilde{\varphi}$. This, however, would require an additional mesh for the Lagrange multiplier field, thus adding complexity to the formulation. Since previous work by Guidault and Belytschko [23] has demonstrated that interpolating the Lagrange multiplier $\phi$ and fine mapping $\widetilde{\boldsymbol{\varphi}}$ with the same basis functions would lead to severe shear locking, we instead interpolate $\phi$ and $\bar{\varphi}$ with the same basis functions such that the finite dimensional formulation can be simplified without introducing shear locking. Also, we concentrate our attention to the overlapped domain $\mathcal{B}^{c}$, in which by virtue of this assumption the number of nodes for the discrete coarse field $\bar{\varphi}_{h}$ and discrete Lagrange multiplier $\boldsymbol{\phi}_{h}$ is the same. Outside the overlapped region, the finite element formulation reduces to a standard one in each of the purely coarse and purely fine domains due to the properties of the weighting functions $\chi$ and $\psi$. Thus, we have,

$\overline{\boldsymbol{\varphi}}_{h}(\boldsymbol{X}):=N_{A}(\boldsymbol{X}) \overline{\boldsymbol{\varphi}}_{A} \in \bar{U}_{h}, \quad \overline{\boldsymbol{\xi}}_{h}(\boldsymbol{X}):=N_{B}(\boldsymbol{X}) \bar{\xi}_{B} \in \bar{U}_{h}$,

$\widetilde{\boldsymbol{\varphi}}_{h}(\boldsymbol{X}):=\lambda_{\alpha}(\boldsymbol{X}) \widetilde{\boldsymbol{\varphi}}_{\alpha} \in \widetilde{U}_{h}, \quad \widetilde{\boldsymbol{\xi}}_{h}(\boldsymbol{X}):=\lambda_{\beta}(\boldsymbol{X}) \widetilde{\boldsymbol{\xi}}_{\beta} \in \widetilde{U}_{h}$,

$\boldsymbol{\phi}_{h}(\boldsymbol{X}):=N_{a}(\boldsymbol{X}) \boldsymbol{\phi}_{a} \in V_{h}, \quad \boldsymbol{\eta}_{h}(\boldsymbol{X}):=N_{b}(\boldsymbol{X}) \boldsymbol{\eta}_{b} \in V_{h}$,

where $\left(N_{A}, N_{B}\right)$ are interpolation functions for $\left(\overline{\boldsymbol{\varphi}}_{h}, \overline{\boldsymbol{\xi}}_{h}\right)$, $\left(\lambda_{\alpha}, \lambda_{\beta}\right)$ are interpolation functions for $\left(\widetilde{\varphi}_{h}, \widetilde{\xi}_{h}\right)$, and $\left(N_{a}, N_{b}\right)$ are interpolation functions for $\left(\boldsymbol{\phi}_{h}, \boldsymbol{\eta}_{h}\right)$ which are the same as for the coarse deformation mapping but limited to the overlapped domain. In addition $(A, B) \in\left[1 \ldots N_{\bar{U}}\right],(\alpha, \beta)$ $\in\left[1 \ldots N_{\widetilde{U}}\right]$ and $(a, b) \in\left[1 \ldots N_{V}\right]$ are indices corresponding to the coarse, fine and overlapped domains with $N_{\bar{U}}$, $N_{\widetilde{U}}$ and $N_{V}$ the number of nodes for their respective domains. Furthermore $\bar{U}_{h} \subset \bar{U}, \widetilde{U}_{h} \subset \widetilde{U}$ and $V_{h} \subset V$ are finite-dimensional subspaces of $\bar{U}, \widetilde{U}$ and $V$ spanned by the corresponding interpolation functions. Introducing this discretization into the variational statement (2.3) gives 
$D \Phi\left[\overline{\boldsymbol{\varphi}}_{h}, \widetilde{\boldsymbol{\varphi}}_{h}, \boldsymbol{\phi}_{h}\right]\left(\overline{\boldsymbol{\xi}}_{h}\right)=\overline{\boldsymbol{R}}_{A}\left[\overline{\boldsymbol{\varphi}}_{h}, \boldsymbol{\phi}_{h}\right] \cdot \overline{\boldsymbol{\xi}}_{A}=0$,

$D \Phi\left[\overline{\boldsymbol{\varphi}}_{h}, \widetilde{\boldsymbol{\varphi}}_{h}, \boldsymbol{\phi}_{h}\right]\left(\widetilde{\boldsymbol{\xi}}_{h}\right)=\widetilde{\boldsymbol{R}}_{\alpha}\left[\widetilde{\boldsymbol{\varphi}}_{h}, \boldsymbol{\phi}_{h}\right] \cdot \widetilde{\boldsymbol{\xi}}_{\alpha}=0$,

$D \Phi\left[\overline{\boldsymbol{\varphi}}_{h}, \widetilde{\boldsymbol{\varphi}}_{h}, \boldsymbol{\phi}_{h}\right]\left(\boldsymbol{\eta}_{h}\right)=\boldsymbol{R}_{a}^{c}\left[\overline{\boldsymbol{\varphi}}_{h}, \widetilde{\boldsymbol{\varphi}}_{h}\right] \cdot \boldsymbol{\eta}_{a}=0$.

where $\overline{\boldsymbol{R}}_{A}, \widetilde{\boldsymbol{R}}_{\alpha}$ and $\boldsymbol{R}_{a}^{c}$ are the residuals of the nonlinear algebraic equations that result from the discrete variational statements (2.8), i.e.,

$$
\begin{aligned}
\overline{\boldsymbol{R}}_{A}\left[\overline{\boldsymbol{\varphi}}_{h}, \boldsymbol{\phi}_{h}\right]= & \int_{\mathcal{B}}\left(\chi \overline{\boldsymbol{P}}: \overline{\mathcal{B}}_{A}-\psi \overline{\boldsymbol{B}} N_{A}\right) d V \\
& +\int_{\mathcal{B}^{c}}\left(\boldsymbol{\phi} N_{A}+\kappa \ell^{2} \operatorname{Grad} \boldsymbol{\phi}: \overline{\mathcal{B}}_{A}\right) d V \\
& -\int_{\partial_{T} \mathcal{B}} \psi \overline{\boldsymbol{T}} N_{A} d S, \\
\widetilde{\boldsymbol{R}}_{\alpha}\left[\widetilde{\boldsymbol{\varphi}}_{h}, \boldsymbol{\phi}_{h}\right]= & \int_{\mathcal{B}}\left[(1-\chi) \widetilde{\boldsymbol{P}}: \widetilde{\mathcal{B}}_{\alpha}-(1-\psi) \widetilde{\boldsymbol{B}} \lambda_{\alpha}\right] d V \\
& -\int_{\mathcal{B}^{c}}\left(\boldsymbol{\phi} \lambda_{\alpha}+\kappa \ell^{2} \operatorname{Grad} \boldsymbol{\phi}: \widetilde{\mathcal{B}}_{\alpha}\right) d V \\
& -\int_{\partial_{T} \mathcal{B}}(1-\psi) \widetilde{\boldsymbol{T}} \cdot \lambda_{\alpha} d S, \\
\boldsymbol{R}_{a}^{c}\left[\overline{\boldsymbol{\varphi}}_{h}, \widetilde{\boldsymbol{\varphi}}_{h}\right]= & \int_{\mathcal{B}^{c}}\left(\overline{\boldsymbol{\varphi}} N_{a}+\kappa \ell^{2} \operatorname{Grad} \overline{\boldsymbol{\varphi}}: \overline{\mathcal{B}}_{a}\right) d V \\
& -\int_{\mathcal{B}^{c}}\left(\widetilde{\boldsymbol{\varphi}} N_{a}+\kappa \ell^{2} \operatorname{Grad} \widetilde{\boldsymbol{\varphi}}: \overline{\mathcal{B}}_{a}\right) d V,
\end{aligned}
$$

where $\overline{\mathcal{B}}_{A}, \widetilde{\mathcal{B}}_{\alpha}$ and $\overline{\mathcal{B}}_{a}$ are third-order tensors that read,

$$
\begin{aligned}
& \overline{\mathcal{B}}_{A}=\delta_{i k} \boldsymbol{N}_{A, j} \boldsymbol{e}_{i} \otimes \boldsymbol{e}_{j} \otimes \boldsymbol{e}_{k}, \\
& \widetilde{\mathcal{B}}_{\alpha}=\delta_{i k} \lambda_{\alpha, j} \boldsymbol{e}_{i} \otimes \boldsymbol{e}_{j} \otimes \boldsymbol{e}_{k}, \\
& \overline{\mathcal{B}}_{a}=\delta_{i k} \boldsymbol{N}_{a, j} \boldsymbol{e}_{i} \otimes \boldsymbol{e}_{j} \otimes \boldsymbol{e}_{k} .
\end{aligned}
$$

The unknowns in the discrete variational statement are the nodal values of $\overline{\boldsymbol{\varphi}}_{A}, \widetilde{\boldsymbol{\varphi}}_{\alpha}$ and $\boldsymbol{\phi}_{a}$. Thus, they constitute a system of nonlinear algebraic equations, i.e., $\overline{\boldsymbol{R}}_{A}\left[\overline{\boldsymbol{\varphi}}_{h}, \boldsymbol{\phi}_{h}\right]$ $=\mathbf{0}, \widetilde{\boldsymbol{R}}_{\alpha}\left[\widetilde{\boldsymbol{\varphi}}_{h}, \boldsymbol{\phi}_{h}\right]=\mathbf{0}$ and $\boldsymbol{R}_{a}^{c}\left[\overline{\boldsymbol{\varphi}}_{h}, \widetilde{\boldsymbol{\varphi}}_{h}\right]=\mathbf{0}$.

\subsection{Linearization}

To obtain solutions from the nonlinear discrete variational statement, one may apply Newton-type methods or other related linearization-based techniques so that incremental solutions can be obtained from the directional derivative of the residuals at each incremental step. Assuming that all loads are conservative, the linear approximation of the residual at $\left(\overline{\boldsymbol{\varphi}}_{n}, \widetilde{\boldsymbol{\varphi}}_{n}, \boldsymbol{\phi}_{n}\right)$ reads

$$
\begin{aligned}
& \overline{\boldsymbol{R}}_{A}\left[\overline{\boldsymbol{\varphi}}_{n+1}, \boldsymbol{\phi}_{n+1}\right] \approx \overline{\boldsymbol{R}}_{A}\left[\overline{\boldsymbol{\varphi}}_{n}, \boldsymbol{\phi}_{n}\right]+D_{\bar{\varphi}} \overline{\boldsymbol{R}}_{A}\left[\overline{\boldsymbol{\varphi}}_{n}, \boldsymbol{\phi}_{n}\right] \\
& \triangle \overline{\boldsymbol{\varphi}}+D_{\boldsymbol{\phi}} \overline{\boldsymbol{R}}_{A}\left[\overline{\boldsymbol{\varphi}}_{n}, \boldsymbol{\phi}_{n}\right] \cdot \Delta \boldsymbol{\phi}, \\
& \widetilde{\boldsymbol{R}}_{\alpha}\left[\widetilde{\boldsymbol{\varphi}}_{n+1}, \boldsymbol{\phi}_{n+1}\right] \approx \widetilde{\boldsymbol{R}}_{\alpha}\left[\widetilde{\boldsymbol{\varphi}}_{n}, \boldsymbol{\phi}_{n}\right]+D_{\widetilde{\boldsymbol{\varphi}}} \widetilde{\boldsymbol{R}}_{\alpha}\left[\widetilde{\boldsymbol{\varphi}}_{n}, \boldsymbol{\phi}_{n}\right] \\
& \triangle \widetilde{\boldsymbol{\varphi}}+D_{\boldsymbol{\phi}} \widetilde{\boldsymbol{R}}_{\alpha}\left[\widetilde{\boldsymbol{\varphi}}_{n}, \boldsymbol{\phi}_{n}\right] \cdot \triangle \boldsymbol{\phi}, \\
& \boldsymbol{R}_{a}^{c}\left[\overline{\boldsymbol{\varphi}}_{n+1}, \widetilde{\boldsymbol{\varphi}}_{n+1}\right] \approx \boldsymbol{R}_{a}^{c}\left[\overline{\boldsymbol{\varphi}}_{n}, \widetilde{\boldsymbol{\varphi}}_{n}\right]+D_{\bar{\varphi}} \boldsymbol{R}_{a}^{c}\left[\overline{\boldsymbol{\varphi}}_{n}, \widetilde{\boldsymbol{\varphi}}_{n}\right] \\
& \triangle \overline{\boldsymbol{\varphi}}+D_{\widetilde{\varphi}} \boldsymbol{R}_{a}^{c}\left[\overline{\boldsymbol{\varphi}}_{n}, \widetilde{\boldsymbol{\varphi}}_{n}\right] \cdot \triangle \widetilde{\boldsymbol{\varphi}}
\end{aligned}
$$

where $\Delta \bar{\varphi}$ and $\Delta \widetilde{\varphi}$ denote the linear incremental displacement from $\overline{\boldsymbol{\varphi}}_{n}(\overline{\mathcal{B}})$ to $\bar{\varphi}_{n+1}(\overline{\mathcal{B}})$, and from $\widetilde{\boldsymbol{\varphi}}_{n}(\widetilde{\mathcal{B}})$ to $\widetilde{\boldsymbol{\varphi}}_{n+1}(\widetilde{\mathcal{B}})$ respectively, and $\triangle \phi$ is the linear increment in the Lagrange multiplier from $\boldsymbol{\phi}_{n}$ to $\boldsymbol{\phi}_{n+1}$. Introducing the discretization (2.7) for the increments, the directional derivatives with respect to $\bar{\varphi}_{n}, \widetilde{\varphi}_{n}$ and $\phi_{n}$ read,

$$
\begin{aligned}
D \overline{\boldsymbol{R}}_{A}\left[\overline{\boldsymbol{\varphi}}_{n}, \boldsymbol{\phi}_{n}\right] \cdot \Delta \overline{\boldsymbol{\varphi}} & =\overline{\boldsymbol{K}}_{A B} \cdot \Delta \overline{\boldsymbol{\varphi}}_{B}, \\
D \widetilde{\boldsymbol{R}}_{\alpha}\left[\widetilde{\boldsymbol{\varphi}}_{n}, \boldsymbol{\phi}_{n}\right] \cdot \Delta \widetilde{\boldsymbol{\varphi}} & =\widetilde{\boldsymbol{K}}_{\alpha \beta} \cdot \Delta \widetilde{\boldsymbol{\varphi}}_{\beta}, \\
D \overline{\boldsymbol{R}}_{A}\left[\overline{\boldsymbol{\varphi}}_{n}, \boldsymbol{\phi}_{n}\right] \cdot \Delta \boldsymbol{\phi} & =\overline{\boldsymbol{C}}_{A b} \cdot \Delta \boldsymbol{\phi}_{b}, \\
D \widetilde{\boldsymbol{R}}_{\alpha}\left[\widetilde{\boldsymbol{\varphi}}_{n}, \boldsymbol{\phi}_{n}\right] \cdot \Delta \boldsymbol{\phi} & =-\widetilde{\boldsymbol{C}}_{\alpha b} \cdot \Delta \boldsymbol{\phi}_{b}, \\
D \boldsymbol{R}_{a}^{c}\left[\overline{\boldsymbol{\varphi}}_{n}, \widetilde{\boldsymbol{\varphi}}_{n}\right] \cdot \Delta \overline{\boldsymbol{\varphi}} & =\overline{\boldsymbol{C}}_{B a}^{T} \cdot \Delta \overline{\boldsymbol{\varphi}}_{B}, \\
D \boldsymbol{R}_{a}^{c}\left[\overline{\boldsymbol{\varphi}}_{n}, \widetilde{\boldsymbol{\varphi}}_{n}\right] \cdot \Delta \widetilde{\boldsymbol{\varphi}} & =-\widetilde{\boldsymbol{C}}_{\beta a}^{T} \cdot \Delta \widetilde{\boldsymbol{\varphi}}_{\beta},
\end{aligned}
$$

where

$$
\begin{aligned}
\overline{\boldsymbol{K}}_{A B} & :=\int_{\mathcal{B}} \chi \overline{\mathcal{B}}_{A}^{T}: \overline{\mathbb{C}}: \overline{\mathcal{B}}_{B} d V, \\
\widetilde{\boldsymbol{K}}_{\alpha \beta} & :=\int_{\mathcal{B}}(1-\chi) \widetilde{\mathcal{B}}_{\alpha}^{T}: \widetilde{\mathbb{C}}: \widetilde{\mathcal{B}}_{\beta} d V, \\
\overline{\boldsymbol{C}}_{A b} & :=\int_{\mathcal{B}^{c}}\left(N_{A} N_{b} \boldsymbol{I}+\kappa \ell^{2} \overline{\mathcal{B}}_{A}^{T}: \overline{\mathcal{B}}_{b}\right) d V, \\
\widetilde{\boldsymbol{C}}_{\alpha b} & :=\int_{\mathcal{B}^{c}}\left(\lambda_{\alpha} N_{b} \boldsymbol{I}+\kappa \ell^{2} \widetilde{\mathcal{B}}_{\alpha}^{T}: \overline{\mathcal{B}}_{b}\right) d V .
\end{aligned}
$$

The two-point tensors $\overline{\mathbb{C}}=\partial^{2} \bar{W} / \partial \overline{\boldsymbol{F}} \partial \overline{\boldsymbol{F}}$ and $\widetilde{\mathbb{C}}=\partial^{2} \widetilde{W} / \partial \widetilde{\boldsymbol{F}} \partial \widetilde{\boldsymbol{F}}$ are fourth-order tensors evaluated in the configurations $\bar{\varphi}_{n}(\overline{\mathcal{B}})$ and $\widetilde{\varphi}_{n}(\widetilde{\mathcal{B}})$ respectively. Hence, the linearized discrete equation (2.11) can be written in a matrix form, i.e.,

$$
\left[\begin{array}{ccc}
\overline{\boldsymbol{K}}_{A B} & \mathbf{0}_{A \beta} & \overline{\boldsymbol{C}}_{A b} \\
\mathbf{0}_{\alpha B} & \widetilde{\boldsymbol{K}}_{\alpha \beta} & -\widetilde{\boldsymbol{C}}_{\alpha b} \\
\overline{\boldsymbol{C}}_{B a}^{T} & -\widetilde{\boldsymbol{C}}_{\beta a}^{T} & \boldsymbol{0}_{a b}
\end{array}\right]\left[\begin{array}{c}
\triangle \overline{\boldsymbol{\varphi}}_{B} \\
\triangle \widetilde{\boldsymbol{\varphi}}_{\beta} \\
\triangle \boldsymbol{\phi}_{b}
\end{array}\right]=\left[\begin{array}{c}
\overline{\boldsymbol{F}}_{A} \\
\widetilde{\boldsymbol{F}}_{\alpha} \\
\mathbf{0}_{a}
\end{array}\right],
$$

where $\overline{\boldsymbol{F}}_{A}=-\overline{\boldsymbol{R}}_{A}\left[\overline{\boldsymbol{\varphi}}_{n}, \boldsymbol{\phi}_{n}\right]$ and $\widetilde{\boldsymbol{F}}_{\alpha}=-\widetilde{\boldsymbol{R}}_{\alpha}\left[\overline{\boldsymbol{\varphi}}_{n}, \widetilde{\boldsymbol{\varphi}}_{n}\right]$. This completes the finite element formulation. 


\section{Numerical stability}

A necessary (but not sufficient) condition to obtain convergent solutions for the nonlinear field equation is that its incremental solution from the linearized system of equations is stable. Stability of the incremental solutions requires fulfilling the coercivity condition. In addition, if solutions are composed of multiple type of fields as expressed in (2.14), then the selected finite dimensional spaces of the trial function and solution fields must also satisfy the inf-sup condition. $[3,5,17,18]$.

The objective of this section is to describe a numerical procedure to verify whether the formulation in Sect. 2.3 is stable for the selected spaces $\bar{U}, \widetilde{U}$ and $V$.

\subsection{Static condensation of the linearized three-field equation}

To simplify the analysis of the inf-sup condition, we first condense the linearized three-field equation (2.14) into a twofield problem. There are two possible ways to condense the three-field problem. The first way is to eliminate the coarse scale incremental displacement $\triangle \bar{\varphi}$ such that

$$
\left[\begin{array}{cc}
\widetilde{\boldsymbol{K}}_{\alpha \beta} & -\widetilde{\boldsymbol{C}}_{\alpha b} \\
-\widetilde{\boldsymbol{C}}_{\beta a}^{T} & \overline{\boldsymbol{C}}_{M a}^{T} \overline{\boldsymbol{K}}_{M N}^{-1} \overline{\boldsymbol{C}}_{N b}
\end{array}\right]\left[\begin{array}{c}
\Delta \widetilde{\boldsymbol{\varphi}}_{\beta} \\
\triangle \boldsymbol{\phi}_{b}
\end{array}\right]=\left[\begin{array}{c}
\widetilde{\boldsymbol{F}}_{\alpha} \\
-\overline{\boldsymbol{C}}_{M a}^{T} \overline{\boldsymbol{K}}_{M B}^{-1} \overline{\boldsymbol{F}}_{B}
\end{array}\right] .
$$

This static condensation procedure is only valid when $\overline{\boldsymbol{K}}_{A B}$ is non-singular. This is true when appropriate boundary conditions are prescribed in the coarse domain such that all rigid body modes are eliminated [20]. Alternatively, one may eliminate the fine incremental displacement field and obtain a two-field linearized field equation which reads

$$
\left[\begin{array}{ll}
\overline{\boldsymbol{K}}_{A B} & \overline{\boldsymbol{C}}_{A b} \\
\overline{\boldsymbol{C}}_{B a}^{T} & \widetilde{\boldsymbol{C}}_{\mu a}^{T} \widetilde{\boldsymbol{K}}_{\mu \nu}^{-1} \widetilde{\boldsymbol{C}}_{\nu b}
\end{array}\right]\left[\begin{array}{c}
\triangle \overline{\boldsymbol{\varphi}}_{B} \\
\Delta \boldsymbol{\phi}_{b}
\end{array}\right]=\left[\begin{array}{c}
\overline{\boldsymbol{F}}_{A} \\
\widetilde{\boldsymbol{C}}_{\mu a}^{T} \widetilde{\boldsymbol{K}}_{\mu \beta}^{-1} \widetilde{\boldsymbol{F}}_{\beta}
\end{array}\right] .
$$

This static condensation procedure requires that $\widetilde{\boldsymbol{K}}_{\alpha \beta}$ be nonsingular. This is true when appropriate boundary conditions are prescribed in the fine domain such that all rigid body modes in the fine domain are eliminated. In the following analysis, we assume that at least one of the static condensation procedures in (3.1) and (3.2) is valid.

\subsection{Discrete inf-sup condition in Euclidean space}

Following the approach in Brezzi and Marini [17], and in the spirit of Bathe [5], Chapelle and Bathe [19]. we present a simple way to verify whether a particular choice of finitedimensional spaces of the configuration mappings $\bar{U}_{h}, \widetilde{U}_{h}$ and Lagrange multiplier $V_{h}$ is stable. As mentioned previously, the test by no means supersedes the analytical proof of the stability of the nonlinear boundary value problem. Instead, our goal here is to provide some practical instrument to determine whether spurious modes exist at each incremental step.

The key step to simplify the analysis is to take advantage of the fact that the spaces $\bar{U}_{h}, \widetilde{U}_{h}$ and $V_{h}$ are all finite dimensional and spanned by $N_{A}, \lambda_{\alpha}$ or $N_{a}$. This is accomplished by passing from these discrete spaces to $\mathbb{R}^{C}, \mathbb{R}^{F}$ or $\mathbb{R}^{L}$, in which $C:=N_{\bar{U}} \times N, F:=N_{\widetilde{U}} \times N$ and $L:=N_{V} \times N$, with $N_{\bar{U}}, N_{\widetilde{U}}$ and $N_{V}$ the number of nodes for $\bar{U}_{h}, \widetilde{U}_{h}$ and $V_{h}$ respectively, and $N$ the spatial dimension. We then may use linear algebra to test whether the inf-sup condition is held for a particular choice of $\bar{U}_{h}, \widetilde{U}_{h}$ and $V_{h}$.

Here we adopt the approach of Brezzi and Marini [17] in which the finite element spaces $\bar{U}, \widetilde{U}$ and $V$ are equipped with the following energy norms,

$$
\begin{aligned}
& \|\bar{\xi}\|_{\bar{U}}^{2}=\int_{\mathcal{B}^{c}} \chi \operatorname{Grad} \bar{\xi}: \overline{\mathbb{C}}: \operatorname{Grad} \bar{\xi} d V, \\
& \|\widetilde{\xi}\|_{\widetilde{U}}^{2}=\int_{\mathcal{B}^{c}}(1-\chi) \operatorname{Grad} \widetilde{\xi}: \widetilde{\mathbb{C}}: \operatorname{Grad} \widetilde{\xi} d V, \\
& \|\eta\|_{V}^{2}=\int_{\mathcal{B}^{c}} \operatorname{Grad} \eta: \overline{\mathbb{C}}^{-1}: \operatorname{Grad} \eta d V .
\end{aligned}
$$

where $\chi \overline{\mathbb{C}}$ and $(1-\chi) \widetilde{\mathbb{C}}$ are strongly elliptic, fourth-order tensors possessing major symmetries and $\chi \in(0,1)$. Note that by definition, norms must be strictly positive. Thus, the strong ellipticity of both $\chi \overline{\mathbb{C}}$ and $(1-\chi) \widetilde{\mathbb{C}}$ are required if the energy norms defined in (3.3) are used to construct the inf-sup test. However, if either $\chi \overline{\mathbb{C}}$ or $(1-\chi) \widetilde{\mathbb{C}}$ becomes singular, then a local loss of coercivity, as defined in Ben Dhia [13], may occur. In order to obtain a stable solution for the linearized field equation (2.14), two inf-sup conditions must be satisfied, i.e.,

$$
\inf _{\eta \in V} \sup _{\overline{\boldsymbol{\xi}} \in \bar{U}} \frac{\int_{\mathcal{B}^{c}}\left(\boldsymbol{\eta} \cdot \overline{\boldsymbol{\xi}}+\kappa \ell^{2} \operatorname{Grad} \boldsymbol{\eta}: \operatorname{Grad} \overline{\boldsymbol{\xi}}\right) d V}{\|\overline{\boldsymbol{\xi}}\|_{\bar{U}}\|\boldsymbol{\eta}\|_{V}} \geq \beta_{1}>0,
$$

and

$$
\inf _{\boldsymbol{\eta} \in V} \sup _{\widetilde{\boldsymbol{\xi}} \in \widetilde{U}} \frac{\int_{\mathcal{B}^{c}} \boldsymbol{\eta} \cdot \widetilde{\boldsymbol{\xi}}+\kappa \ell^{2} \operatorname{Grad} \eta: \operatorname{Grad} \widetilde{\boldsymbol{\xi}} d V}{\|\widetilde{\boldsymbol{\xi}}\|_{\widetilde{U}}\|\boldsymbol{\eta}\|_{V}} \geq \beta_{2}>0,
$$

where $\beta_{1}$ and $\beta_{2}$ are positive constants. Now, notice that we may construct a bijective map between $\bar{U}_{h}$ and the Euclidean space $\mathbb{R}^{C}$ as

$\bar{\delta}:=\left(\overline{\boldsymbol{\delta}}_{1}, \ldots \overline{\boldsymbol{\delta}}_{A}, \ldots \overline{\boldsymbol{\delta}}_{N_{\bar{U}}}\right)$,

where $\bar{\delta} \in \mathbb{R}^{C}$. Similarly, a bijective map can be constructed between $\widetilde{U}_{h}$ and $\mathbb{R}^{F}$,

$\widetilde{\boldsymbol{\delta}}:=\left(\widetilde{\boldsymbol{\delta}}_{1}, \ldots \widetilde{\boldsymbol{\delta}}_{\alpha}, \ldots \widetilde{\boldsymbol{\delta}}_{N_{\tilde{U}}}\right)$, 
where $\delta \in \mathbb{R}^{F}$. A bijective map can be constructed as well between $V_{h}$ and $\mathbb{R}^{L}$ as

$\boldsymbol{\theta}:=\left(\boldsymbol{\theta}_{1}, \ldots \boldsymbol{\theta}_{a}, \ldots \boldsymbol{\theta}_{N_{V}}\right)$,

in which $\boldsymbol{\theta} \in \mathbb{R}^{L}$. By replacing the function spaces $\bar{U}, \widetilde{U}$ and $V$ with the Euclidean spaces $\mathbb{R}^{C}, \mathbb{R}^{F}$ and $\mathbb{R}^{L}$, the infsup condition expressed in (3.4) and (3.5) can be rewritten in terms of vectors and matrices as

$$
\inf _{\boldsymbol{\theta} \in \mathbb{R}^{L}} \sup _{\bar{\delta} \neq \mathbf{0}} \frac{\boldsymbol{\theta}_{a} \overline{\boldsymbol{C}}_{B a}^{T} \overline{\boldsymbol{\delta}}_{B}}{\left(\boldsymbol{\theta}_{m} \overline{\boldsymbol{Q}}_{m n} \boldsymbol{\theta}_{n}\right)^{\frac{1}{2}}\left(\overline{\boldsymbol{\delta}}_{R} \overline{\boldsymbol{K}}_{R S} \overline{\boldsymbol{\delta}}_{S}\right)^{\frac{1}{2}}} \geq \beta_{1}>0
$$

and

$$
\inf _{\boldsymbol{\theta} \in \mathbb{R}^{L}} \sup _{\widetilde{\delta} \neq \mathbf{0}} \frac{\boldsymbol{\theta}_{a} \widetilde{\boldsymbol{C}}_{\beta a}^{T} \widetilde{\boldsymbol{\delta}}_{\beta}}{\left(\boldsymbol{\theta}_{m} \overline{\boldsymbol{Q}}_{m n} \boldsymbol{\theta}_{n}\right)^{\frac{1}{2}}\left(\widetilde{\boldsymbol{\delta}}_{\rho} \widetilde{\boldsymbol{K}}_{\rho \sigma} \widetilde{\boldsymbol{\delta}}_{\sigma}\right)^{\frac{1}{2}}} \geq \beta_{2}>0,
$$

where the matrix $\overline{\boldsymbol{Q}}_{a b}$ corresponds to the last energy norm defined in (3.3), i.e.,

$\overline{\boldsymbol{Q}}_{a b}:=\int_{\mathcal{B}^{c}} \overline{\mathcal{B}}_{a}^{T}: \overline{\mathbb{C}}^{-1}: \overline{\mathcal{B}}_{b} d V$.

\subsection{Discrete inf-sup test in Euclidean space}

Once the inf-sup condition is expressed in Euclidean space, we may determine the existence of $\beta_{1}$ defined in (3.9). A similar approach was previously used in Brezzi and Marini [17] to derive inf-sup tests for a class of mortar finite element formulations. First, we re-express the supremum in (3.9) by changing the basis vectors such that

$$
\begin{aligned}
S(\overline{\boldsymbol{\delta}}) & =\sup _{\overline{\boldsymbol{\delta}} \neq 0} \frac{\boldsymbol{\theta}_{a} \overline{\boldsymbol{C}}_{B a}^{T} \overline{\boldsymbol{\delta}}_{B}}{\left(\overline{\boldsymbol{\delta}}_{M} \overline{\boldsymbol{K}}_{M N} \overline{\boldsymbol{\delta}}_{N}\right)^{\frac{1}{2}}} \\
& =\sup _{\boldsymbol{z} \neq 0} \frac{\boldsymbol{\theta}_{a} \overline{\boldsymbol{C}}_{M a}^{T}\left(\overline{\boldsymbol{K}}_{M B}\right)^{-\frac{1}{2}} \boldsymbol{z}_{B}}{\left(\boldsymbol{z}_{S} \boldsymbol{z}_{S}\right)^{\frac{1}{2}}},
\end{aligned}
$$

where $\boldsymbol{z}_{A}:=\overline{\boldsymbol{K}}_{A B}^{\frac{1}{2}} \overline{\boldsymbol{\delta}}_{B}$ and $\operatorname{span}(\boldsymbol{z})=\operatorname{span}(\overline{\boldsymbol{\delta}})=\mathbb{R}^{C}$. Note that the supremum is located at $\boldsymbol{z}_{A}=\overline{\boldsymbol{K}}_{A M}^{\frac{1}{2}} \overline{\boldsymbol{C}}_{M b} \boldsymbol{\theta}_{b}$, i.e.,

$S(\overline{\boldsymbol{\delta}})=\left(\boldsymbol{\theta}_{a} \overline{\boldsymbol{C}}_{M a}^{T} \overline{\boldsymbol{K}}_{M N}^{-1} \overline{\boldsymbol{C}}_{N b} \boldsymbol{\theta}_{b}\right)^{\frac{1}{2}}$,

which can be proved by finding the root of

$$
\frac{d}{d z_{R}} \frac{\boldsymbol{\theta}_{a} \overline{\boldsymbol{C}}_{M a}^{T}\left(\overline{\boldsymbol{K}}_{M B}\right)^{-\frac{1}{2}} \boldsymbol{z}_{B}}{\left(\boldsymbol{z}_{S} \boldsymbol{z}_{S}\right)^{\frac{1}{2}}}=\mathbf{0}
$$

By substituting (3.13) into the discrete inf-sup condition (3.9) and squaring both sides, we rewrite the inf-sup condition (3.9) as

$$
\boldsymbol{\theta}_{a} \overline{\boldsymbol{C}}_{M a}^{T} \overline{\boldsymbol{K}}_{M N}^{-1} \overline{\boldsymbol{C}}_{N b} \boldsymbol{\theta}_{b} \geq \beta_{1}^{2} \boldsymbol{\theta}_{a} \overline{\boldsymbol{Q}}_{a b} \boldsymbol{\theta}_{b} \quad \forall \boldsymbol{\theta} \in \mathbb{R}^{L},
$$

The existence of the positive constant $\beta_{1}$ is therefore guaranteed if the smallest eigenvalue of the following generalized eigenvalue problem is larger than zero,

$$
\left(\overline{\boldsymbol{C}}_{M a}^{T} \overline{\boldsymbol{K}}_{M N}^{-1} \overline{\boldsymbol{C}}_{N b}-\bar{\lambda} \overline{\boldsymbol{Q}}_{a b}\right) \boldsymbol{\theta}_{b}=\mathbf{0},
$$

where it is trivial to show that $\bar{\lambda}$ is in fact the largest possible value for $\beta_{1}$ such that the inequality expressed in (3.16) is satisfied.

The eigenvalue problem corresponding to the existence of $\beta_{2}$ can be obtained via a similar procedure. The resultant generalized eigenvalue problem reads

$$
\left(\widetilde{\boldsymbol{C}}_{\alpha a}^{T} \widetilde{\boldsymbol{K}}_{\alpha \beta}^{-1} \widetilde{\boldsymbol{C}}_{\beta b}-\tilde{\lambda} \overline{\boldsymbol{Q}}_{a b}\right) \boldsymbol{\theta}_{b}=\mathbf{0} .
$$

Note that both inf-sup tests (3.16) and (3.17) can be easily generalized for different normed spaces chosen for the Lagrange multiplier. The only required change is to replace the $\bar{Q}$ matrix by a new matrix corresponding to the respective norm.

\section{Nonlocal regularization at the fine scale}

Due to loss of ellipticity, the partial differential equation (2.5) may exhibit mesh dependence when used in conjunction with constitutive modes that admit softening. This non-physical dependence on mesh size can be alleviated by introducing a length scale through regularization procedures [8, 16,22,38]. Here we adopt a variational approach proposed by Mota et al. [29] to regularize (2.5) in the fine domain and use numerical examples to determine whether this is sufficient to alleviate the pathological mesh dependence.

The method described in Mota et al. [29] proposes a variational principle that results in a projection of the internal variable field $\boldsymbol{Z}$ that leads to bifurcation onto patches or subdomains $D$ of equal volume in which the resultant field $\bar{Z}$ is constant, i.e.,

$$
\begin{aligned}
& \bar{Z}=\frac{1}{\operatorname{vol}(D)} \int_{D} \boldsymbol{Z} d V, \\
& \operatorname{vol}(D):=\int_{D} d V .
\end{aligned}
$$

The nonlocal domains $D$ are non-overlapped partitions of the fine domain which define the length scale of the problem. The history-dependent behavior in the fine domain is then computed with the nonlocal $\bar{Z}$ instead of $\boldsymbol{Z}$. 


\section{Block solver for the overlapped domain problem}

The linear system of equations in (2.14) may be illconditioned if the components of $\overline{\boldsymbol{K}}$ and $\widetilde{\boldsymbol{K}}$ are of much larger magnitude than those of $\overline{\boldsymbol{C}}$ and $\widetilde{\boldsymbol{C}}$. In that case, an iterative solver may fail to converge even though a unique solution does exist. Furthermore, the resultant block matrix system can be significantly more expensive to solve if a large portion of the domain is overlapped. The efficiency and accuracy of the solution scheme are improved by the introduction of a simple algorithm designed specifically for the overlapped domain problem.

\subsection{Decoupled algorithm}

Note that (2.14) can be solved sequentially if the numerical values of the Lagrange multiplier are known. To illustrate this point let us rewrite the global linear system of equations corresponding to (2.14) as

$$
\left[\begin{array}{cc}
\boldsymbol{A} & \boldsymbol{B} \\
\boldsymbol{B}^{T} & \mathbf{0}
\end{array}\right]\left[\begin{array}{c}
\triangle \boldsymbol{\varphi} \\
\triangle \boldsymbol{\phi}
\end{array}\right]=\left[\begin{array}{c}
\boldsymbol{F} \\
\mathbf{0}
\end{array}\right],
$$

where matrices $\boldsymbol{A}$ and $\boldsymbol{B}$ are defined as

$$
\boldsymbol{A}=\left[\begin{array}{cc}
\bar{K} & \mathbf{0} \\
\mathbf{0} & \widetilde{\boldsymbol{K}}
\end{array}\right], \quad \boldsymbol{B}=\left[\begin{array}{c}
\overline{\boldsymbol{C}} \\
-\widetilde{C}
\end{array}\right],
$$

where $\overline{\boldsymbol{K}}, \widetilde{\boldsymbol{K}}, \overline{\boldsymbol{C}}$ and $\widetilde{\boldsymbol{C}}$ are the global matrices corresponding to (2.11). The vectors $\triangle \boldsymbol{\varphi}$ and $\boldsymbol{F}$ are defined as

$\triangle \varphi=\left[\begin{array}{c}\triangle \bar{\varphi} \\ \triangle \widetilde{\varphi}\end{array}\right], \quad \boldsymbol{F}=\left[\begin{array}{c}\bar{F} \\ \widetilde{F}\end{array}\right]$,

in which $\triangle \overline{\boldsymbol{\varphi}}, \triangle \widetilde{\boldsymbol{\varphi}}, \overline{\boldsymbol{F}}$ and $\widetilde{\boldsymbol{F}}$ are the global vectors corresponding to (2.11). One important observation here is that $\boldsymbol{A}$ is a block diagonal matrix. This is true even when there are multiple coarse and fine domains in the system. Hence, one may use static condensation in (5.1) to eliminate the displacement increment $\Delta \boldsymbol{\varphi}$ and decouple the block system into the following form

$\triangle \varphi=A^{-1}(\boldsymbol{F}-B \triangle \phi)$

and

$\triangle \boldsymbol{\phi}=\left(\boldsymbol{B}^{T} \boldsymbol{A}^{-1} \boldsymbol{B}\right)^{-1} \boldsymbol{B}^{T} \boldsymbol{A}^{-1} \boldsymbol{F}$.

The resultant system of equations allows each domain to be solved in parallel, since $\boldsymbol{A}$ is block diagonal. Evidently, one does not directly compute the inverses of $\boldsymbol{A}$ or $\boldsymbol{B}^{T} \boldsymbol{A}^{-1} \boldsymbol{B}$ to solve (5.4) and (5.5), but rather uses iterative schemes or special factorizations and the like to solve them.
5.2 Sequential solver for the concurrent coupling problem

Equations (5.4) and (5.5) may be solved with a threestep algorithm in which one first computes the decoupled predictor incremental displacement field $\Delta \boldsymbol{\varphi}_{\text {pred }}:=$ $\boldsymbol{A}^{-1} \boldsymbol{F}$, followed by the computation of the incremental Lagrange multiplier $\Delta \phi$ via (5.5) and the corrected displacement field $\Delta \varphi=\Delta \varphi_{\text {pred }}+\Delta \varphi_{\text {corr }}$ with $\Delta \varphi_{\text {corr }}:=$ $-\boldsymbol{A}^{-1} \boldsymbol{B} \triangle \boldsymbol{\phi}$.

Since $\boldsymbol{A}$ is a block diagonal matrix, the decoupled fine and coarse incremental displacements can be solved for independently to further improve efficiency. The resultant procedure is summarized in Algorithm 1 in which we update the solution by solving two smaller and well-conditioned systems twice instead of attempting to seek the solution of a larger, ill-conditioned block system. This block system could also be solved by a block-preconditioned Newton-Krylov scheme (see Toh et al. [35] White and Borja [36]).

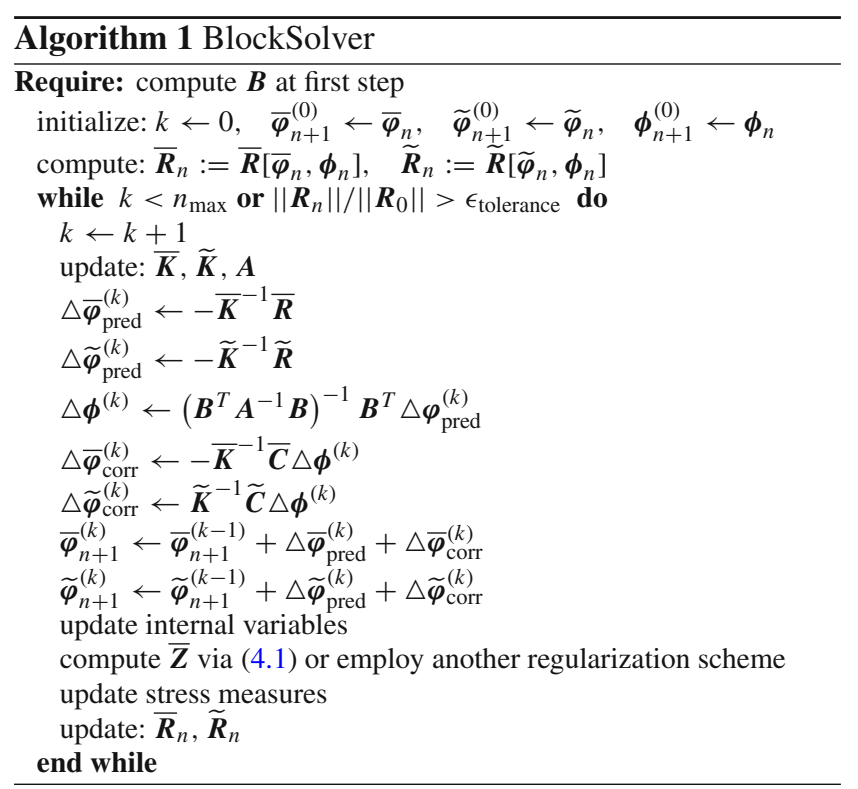

\section{Numerical examples}

This section illustrates both the accuracy and efficiency of the proposed multiscale method. Three numerical examples are examined: the first is a one-dimensional patch test. We use this example to explore the conditions under which the domain coupling method fails to reproduce a linear displacement field. The second example is adopted from Ben Dhia and Rateau [14], Guidault and Belytschko [23] in which a one-dimensional bar is deformed due to self-weight. In the third example, a conical-shapped bar is loaded by prescribing non-zero displacement at the base and fixed at the tip. 


\subsection{One-dimensional patch test}

It is essential for domain coupling methods to pass the patch test [4]. In other words, the overlapped problem is required to reproduce an exact linear displacement field when the analytical solution is linear. A failed patch test indicates that solutions obtained by the corresponding methods are not reliable and may contain spurious information due to numerical deficiencies.

A one-dimensional patch test can be generated by applying prescribed displacements on both ends of a domain with a uniform cross section area and no body force. The solution should result in a linear displacement field and homogeneous strain field [4]. The setup is as follows: let $\mathcal{B}=[0,1], \widetilde{\mathcal{B}}=[0,2 / 3]$ and $\overline{\mathcal{B}}=[1 / 3,1]$. Let the displacements $u(0)=0$ and $u(1)=1$ and the elastic modulus and cross section area be both 1 . The analytical solution is $u(x)=x$.

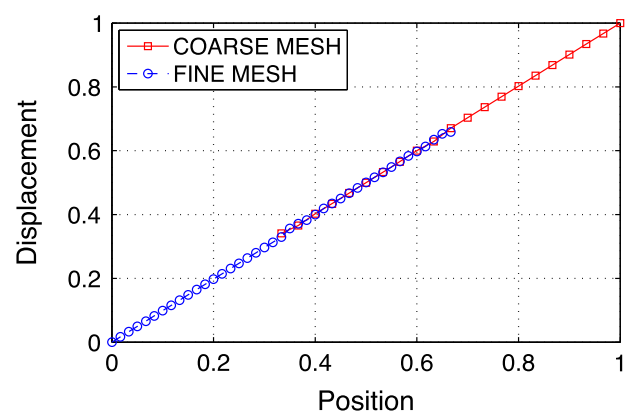

First we turn our attention to the case in which a constant weighting function is used, i.e.,

$\chi(\boldsymbol{X})= \begin{cases}1, & \boldsymbol{X} \in \overline{\mathcal{B}} \backslash \mathcal{B}^{c} ; \\ \frac{1}{2}, & \boldsymbol{X} \in \mathcal{B}^{c} ; \\ 0, & \boldsymbol{X} \in \widetilde{\mathcal{B}} \backslash \mathcal{B}^{c} .\end{cases}$

As a result, the elastic strain energy is equally partitioned in the coarse and fine domains. The size ratio between the coarse and fine elements is $h^{\mathrm{c}} / h^{\mathrm{f}}=2$. Figure 2 a shows the displacement, strain and Lagrange multiplier obtained via $L_{2}$ coupling and the constant weighting function. Note that while the displacement fields of the coarse and fine domains appear to be stable, we observe spurious oscillations in both the strain and Lagrange multiplier. This result is consistent with the findings in [23] which show that the domain coupling method using a constant weighting function and $L_{2}$ coupling is unstable. By contrast, Fig. 2b shows the dis-
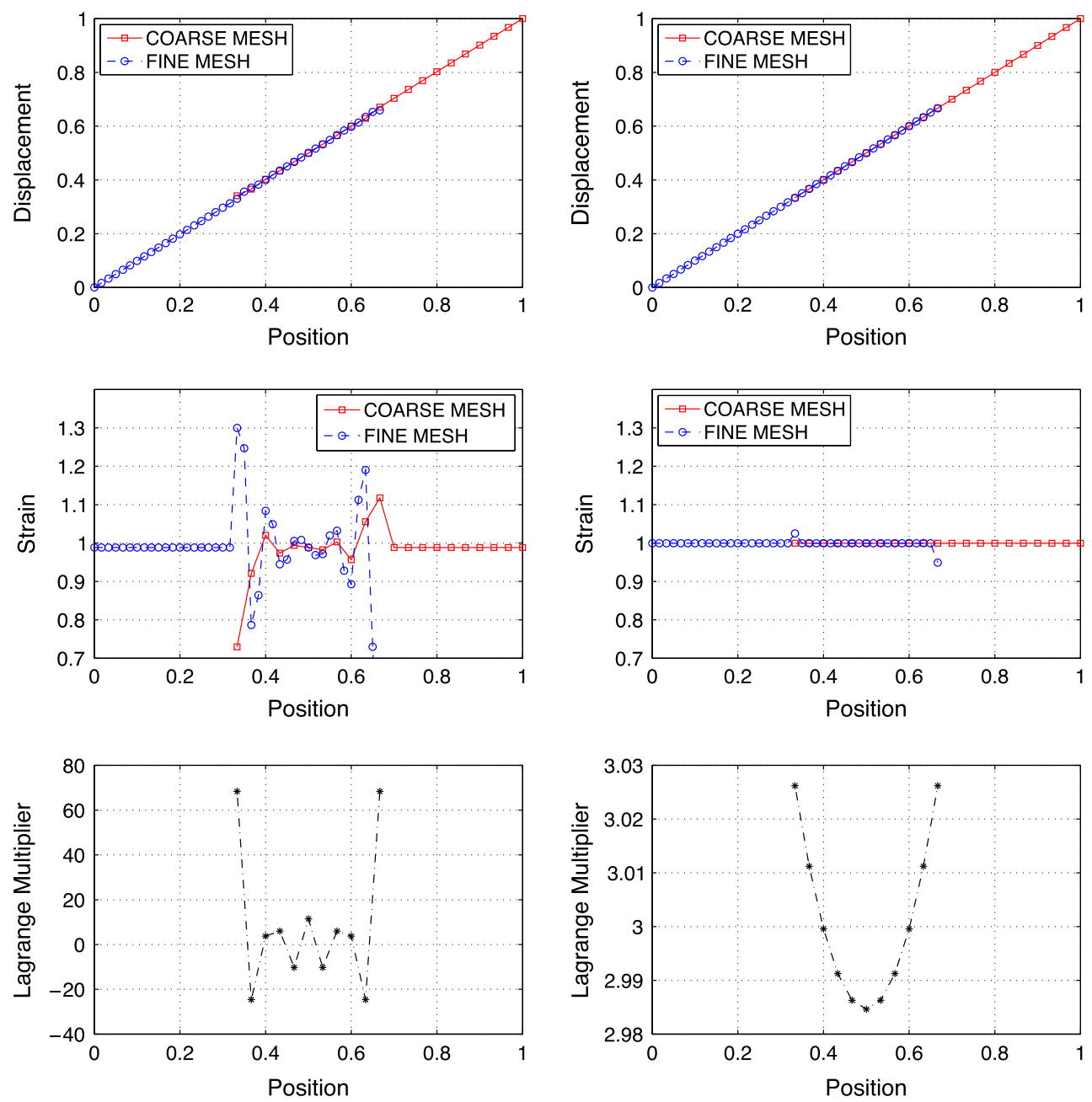

(a) $L_{2}$ coupling

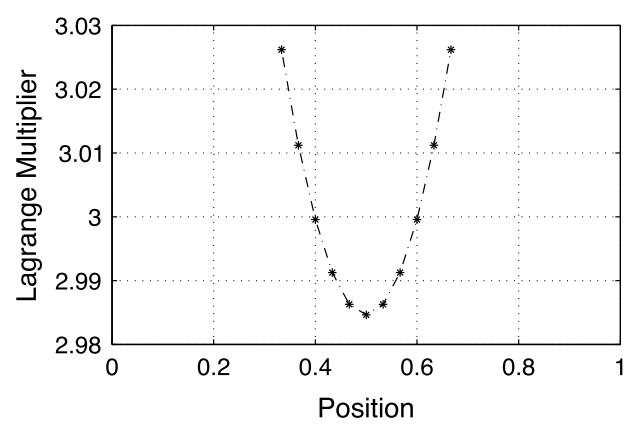

(b) $H^{1}$ coupling

Fig. 2 1D patch test. Displacement, strain and Lagrange multiplier for constant weighting function (6.1) 

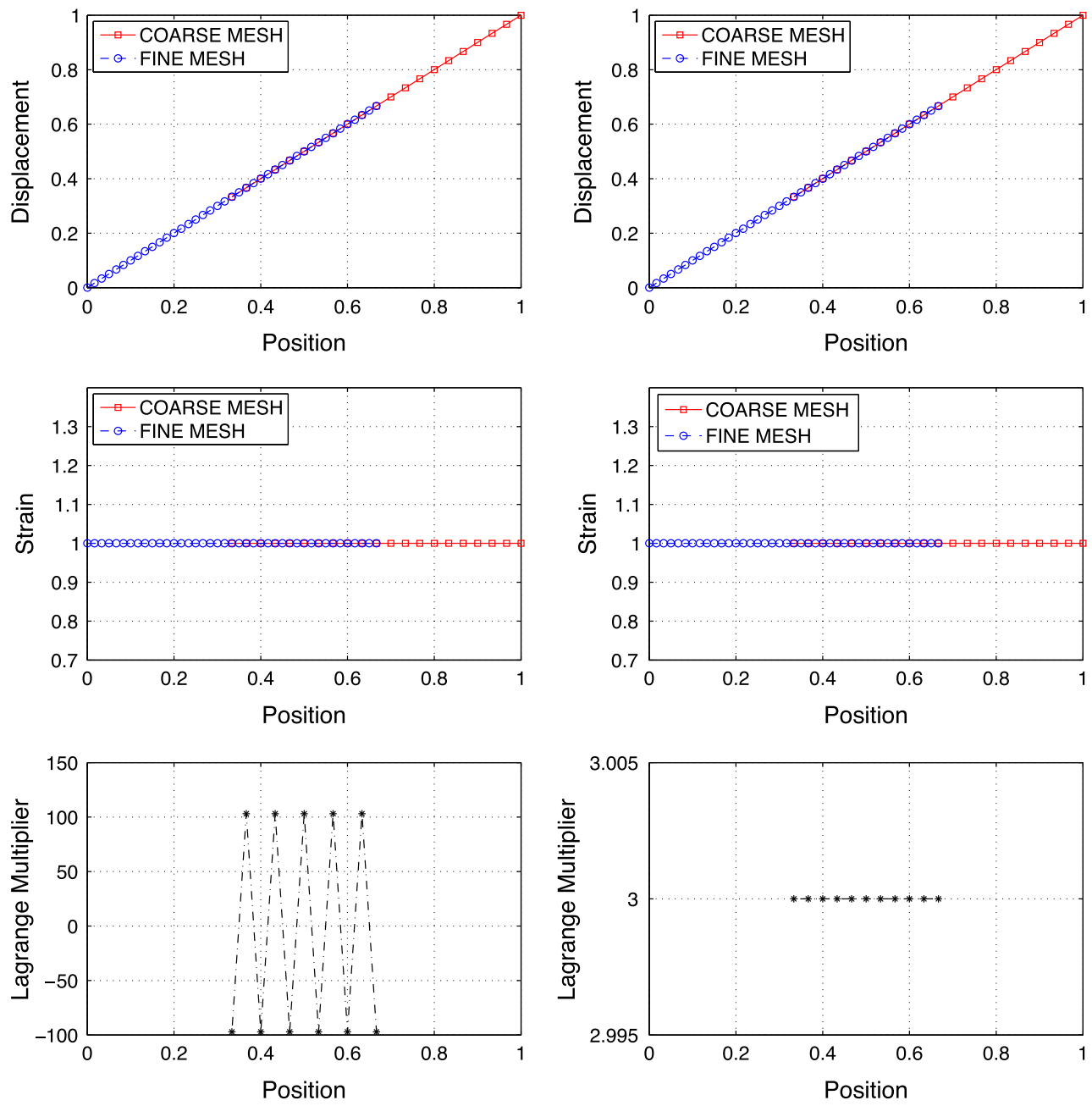

(a) $L_{2}$ coupling

(b) $H^{1}$ coupling

Fig. 3 1D patch test. Displacement, strain and Lagrange multiplier for linear weighting function (6.2)

placement, strain and Lagrange multiplier field obtained by $H^{1}$ coupling, which is enabled by setting $\kappa \ell^{2}=1$. The results shown in Fig. 2b illustrate that $H^{1}$ coupling leads to compatible displacements and a stable Lagrange multiplier in the overlapped region. The oscillations in the strain are less severe as compared to the $L_{2}$ coupling results shown in Fig. 2b, although there are two small spikes in the strain at the boundary of the coupling region.

Next, we repeat the calculation by replacing the constant weighting function with a linear weighting function which reads

$\chi(\boldsymbol{X})= \begin{cases}1, & \boldsymbol{X} \in \overline{\mathcal{B}} \backslash \mathcal{B}^{c} ; \\ 3 x-1, & \boldsymbol{X} \in \mathcal{B}^{c} ; \\ 0, & \boldsymbol{X} \in \widetilde{\mathcal{B}} \backslash \mathcal{B}^{c} .\end{cases}$

Figure $3 \mathrm{a}$, b show the results obtained via $L_{2}$ and $H^{1}$ couplings with this linear weighting function. Both $L_{2}$ and $H^{1}$ coupling methods pass the patch test in the sense that both are able to reproduce a homogeneous strain field. Nevertheless, while the $H^{1}$ coupling method produces a stable Lagrange multiplier field, the $L_{2}$ coupling method fails to do so. Results from this patch test suggests that the $H^{1}$ coupling with a linear weighting function is the most desirable choice for the coupling domain, as it passes the patch test while maintaining stability of the results.

\subsection{Clamped bar loaded by self weight}

We now use a one-dimensional small strain linear elasticity problem to verify our implementation and analyze the stability of the overlapped domain method. This example has been previously studied by Ben Dhia and Rateau [14] to illustrate the relationship between the coupling operator and the deterioration of the condition number. Guidault and Belytschko [23] further analyze how the continuity of the 
Fig. 4 One-dimensional self-weighted bar problem (left) with refinement on fine domain (middle) and both fine and coarse domains (right)
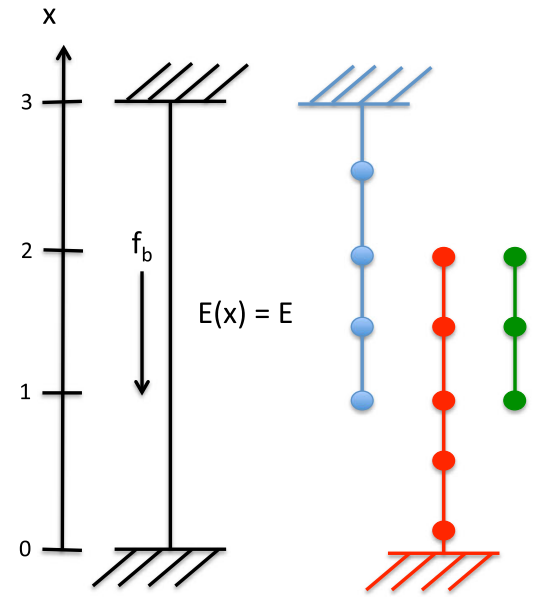

uniform mesh
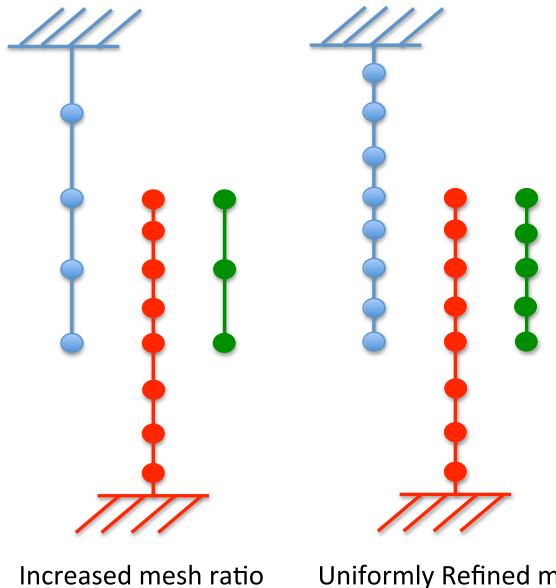

Uniformly Refined mesh

coarse displacement

fine displacement

Lagrange multiplier weighting function $\chi$ affects the stability of the $H^{1}$ and $L_{2}$ couplings.

Consider a one-dimensional vertical bar with uniform cross section which is clamped at the top and the bottom. Both the elastic modulus $E$ and the body force of the bar $f_{b}$ are constant in the domain such that the exact solution of the displacement field reads

$u=\frac{f_{b}}{2 E}(x-L) x$

where $L$ is the length of the bar. In this numerical example, we set $L=3$ and $f_{b} / 2 E=1$. We also set $\kappa \ell^{2}=1$ and $\kappa=0$ for $H^{1}$ and $L_{2}$ couplings, respectively.

Let the mechanical response of both the coarse and fine domains be governed by the same linear elastic constitutive law. Furthermore, we define the coarse domain as $\overline{\mathcal{B}}=[0,2]$ and the fine domain as $\widetilde{\mathcal{B}}=[1,3]$, while choosing a constant weighting function within the overlapped domain, $\chi=0.5$ on $[1,2]$ as depicted in Fig. 4. In these calculations, a onepoint quadrature rule is used to perform the numerical integration.

\subsubsection{Numerical inf-sup test}

Previous works such as Ben Dhia and Rateau [14] and Guidault and Belytschko [23] have demonstrated the difference in stability of the $H^{1}$ and $L_{2}$ coupling methods through numerical examples. On the other hand, the origin of spurious oscillations in the Lagrange multiplier field has been explained in terms of the attainment (or lack thereof) of the inf-sup condition in Bauman et al. [6]. Prudhomme et al. [30] investigate the fulfillment of the inf-sup condition for particle-continuum models and, in fact, show that the infsup constant tends to zero for the $L_{2}$ coupling case. Here our goal is to adopt inf-sup tests to check whether the infsup condition has been attained for a given domain coupling problem.

In this particular example, we use the two inf-sup tests developed in Sect. 3 to investigate the origin of these oscillations. The tests are conducted as follows: first, we compute the matrices $\widetilde{\boldsymbol{C}}, \overline{\boldsymbol{C}}, \widetilde{\boldsymbol{K}}, \overline{\boldsymbol{K}}$ and $\overline{\boldsymbol{Q}}$; then we solve the two generalized eigenvalue problems as defined in (3.16) and (3.17). In the first test (3.16), the existence of a positive inf-sup value $\beta_{1}$ is guaranteed if the smallest eigenvalue remains positive and does not reach zero during refinement.

Figure 5 shows the smallest eigenvalue of the generalized eigenvalue problem (3.16) where we denote the number of coarse elements as $N_{\mathrm{c}}$. We find that for $L_{2}$ coupling the smallest eigenvalue oscillates around zero within machine precision. On the other hand, although the eigenvalue corresponding to $H^{1}$ coupling decreases as the mesh becomes finer, it remains positive and does not vanish. These results show that the statically condensed problem in (3.1) is unstable if $L_{2}$ coupling is used to enforce compatibility.

In the second test defined by (3.17), the smallest eigenvalue is affected by both the level of refinement and the mesh ratio between the coarse and fine meshes. Hence, we compute the smallest eigenvalue corresponding to a wide range of mesh ratios and levels of refinement to ensure the existence of a positive inf-sup value $\beta_{2}$.

Figure 6 compares the smallest eigenvalue of (3.17) of the overlapped domain problem with $H^{1}$ and $L_{2}$ couplings. Again, for $L_{2}$ coupling the smallest eigenvalue is close to zero within machine precision and also oscillating between 


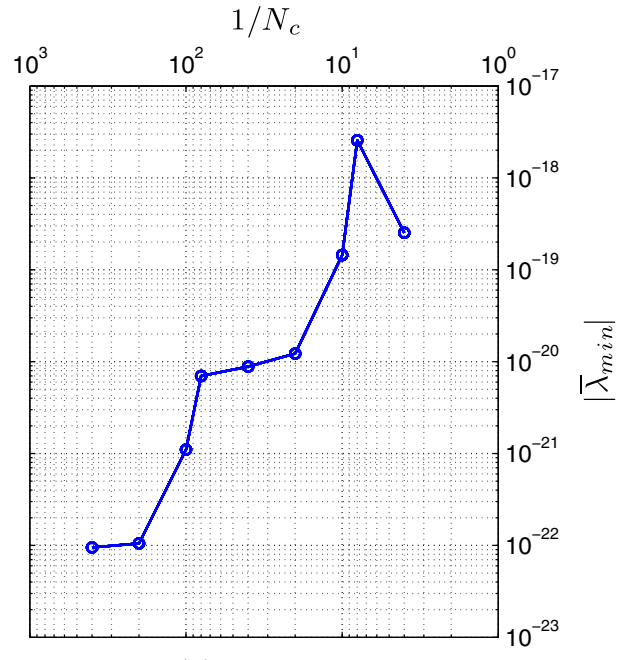

(a) $L_{2}$ coupling

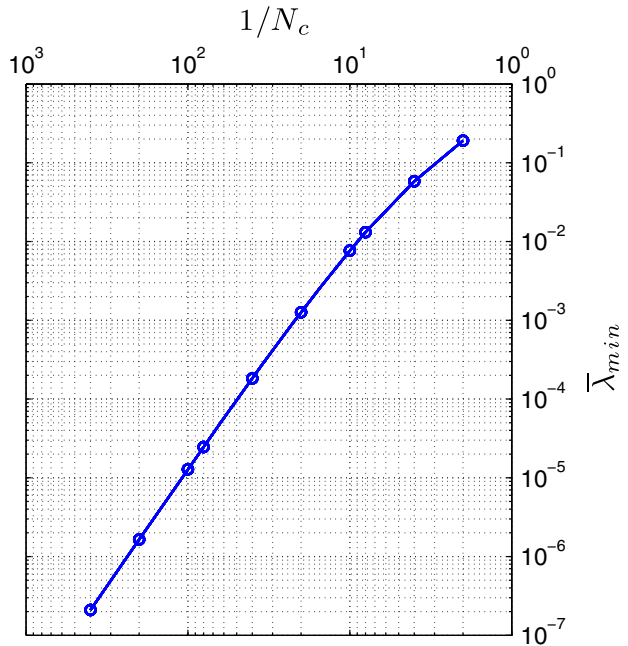

(b) $H^{1}$ coupling

Fig. 5 Clamped bar loaded by self weight. Upper bound of inf-sup value $\beta_{1}$

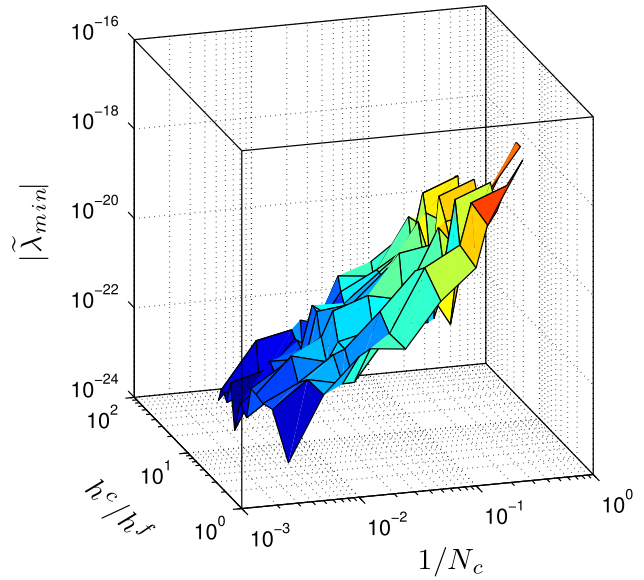

(a) $L_{2}$ coupling

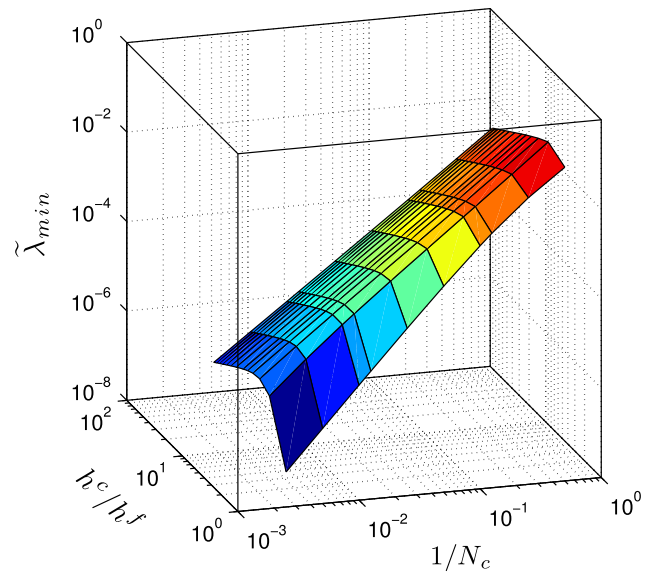

(b) $H^{1}$ coupling

Fig. 6 Clamped bar loaded by self weight. Upper bound of inf-sup value $\beta_{2}$

positive and negative for various combinations of mesh ratios and mesh sizes. This result clearly shows that using $L_{2}$ coupling may lead to a lack of the inf-sup condition. For $H^{1}$ coupling, on the other hand, we observe that while increasing mesh refinement and/or mesh ratio may decrease the upper bound value of the inf-sup value $\beta_{2}$, the smallest eigenvalue remains positive and the change with respect to mesh size is stable.

Results from both inf-sup tests indicate that $H^{1}$ coupling is a more reliable way to enforce weak compatibility if the Lagrange multiplier and the coarse deformation mapping share the same discretization. Although it is possible to develop stabilization procedures to eliminate the oscillation modes in the $L_{2}$ coupling formulation, these numerical treatments are out of the scope of this work.

\subsubsection{Conditioning of the domain coupling method}

The domain coupling method requires two additional parameters: the weighting function $\chi(\boldsymbol{X})$, which determines how the two energy density functions are partitioned, and the length scale parameter $\kappa \ell^{2}$, which determines the significance of the displacement gradient compatibility in minimizing the compatibility energy functional.

Guidault and Belytschko [23] conducted numerical experiments on this self-weighted clamped bar problem to analyze how different choices of Lagrange multiplier space affect the kinematic compatibility and whether they lead to shear locking in the overlapped domain. For a fixed length scale parameter $\kappa \ell^{2}$, they found that $H^{1}$ coupling may lead to fine and coarse displacement fields that lack compatibility within 


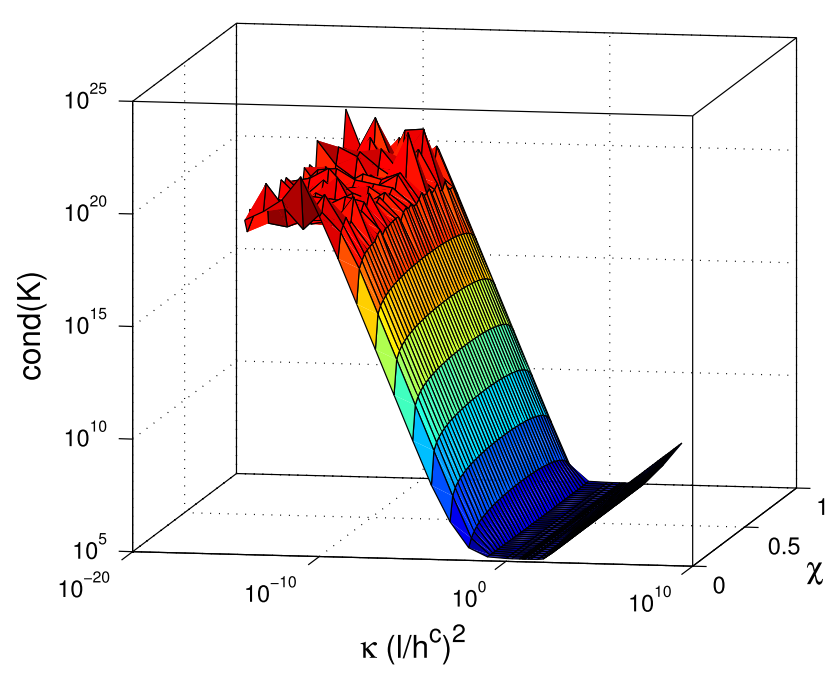

Fig. 7 Clamped bar loaded by self weight. Condition number cond $(\boldsymbol{K})$ with constant weighting function $\chi$ ranging from 0 to $1, \kappa\left(\ell / h^{\mathrm{c}}\right)^{2}$ ranging from $10^{-15}$ to $10^{5}$

the overlapped domain when their mesh sizes are of similar order, while it is beneficial for regions that couple two meshes with very different mesh sizes.

Here our objective is to analyze how varying the length scale parameter $\kappa \ell^{2}$ and the weighting function $\chi$ affect the numerical performance. As mentioned previously, our focus is on the specific case in which the coarse displacement and Lagrange multiplier fields are spanned by the same set of basis functions.

Figure 7 shows the condition number cond $(\boldsymbol{K})$ that results from various values of $\chi$ and $\kappa \ell^{2}$. We notice that the condition number is insensitive to the value of the weighting function, but is strongly dependent on the length scale parameter $\kappa \ell^{2}$. We find that the condition number of the linear system of equations reaches a minimum when $\kappa \ell^{2}=\left(h^{\mathrm{c}}\right)^{2}$.

\subsubsection{Parametric effects on stability and accuracy}

Next, we compare the solution fields obtained by setting $\kappa \ell^{2}=\left(h^{\mathrm{c}}\right)^{2}$ with those obtained with $L_{2}$ coupling $(\kappa=0)$. Figure 8a-f compare the displacement fields obtained from $L_{2}$ and $H^{1}$ couplings with constant weighting function and various mesh ratios. The results indicate that $L_{2}$ coupling performs well if $h^{\mathrm{f}}=h^{\mathrm{c}}$, but may introduce spurious oscillations if the mesh ratio $h^{\mathrm{c}} / h^{\mathrm{f}}$ is higher. This is due to the fact that compatibility is only enforced weakly and that no gradient control is applied in this case.

Figure $8 b, d, f$ show the displacement field obtained with $H^{1}$ coupling. In contrast to the $L_{2}$ results shown in Fig. 8a, c, $\mathrm{e}$, the coarse and fine displacement fields are more compatible when the mesh ratio is higher. In all three simulations, $H^{1}$ coupling does not exhibit any spurious oscillations in either the fine or coarse displacement fields.

\subsection{Singular bar}

Consider a one-dimensional bar with cross section area $A(X)=A_{0} \sqrt{X}$ clamped at $X=0$ and with a prescribed displacement $d_{0}$ at the end $X=L$. For a linear elastic material undergoing small deformations, the analytical solution of this problem reads

$u(X)=d_{0} \sqrt{\frac{X}{L}}$.

Note that the origin $X=0$ is a singular point for the strain that mimics the behavior of a sharp crack in linear elastic fracture mechanics.

We solve this problem with $L=1$ and $E A_{0}=1$. The overlapped domain is located at $X \in\left[\frac{1}{3}, \frac{2}{3}\right]$ and the coarse and fine strain energy densities are evenly partitioned in the coupling region. Figure 9 shows a series of finite element solutions for the same coarse mesh in $X \in\left[0, \frac{2}{3}\right]$ and with fine meshes in $X \in\left[\frac{1}{3}, 1\right]$ with various levels of refinement that converge to the analytical solution.

We examine this problem further by using now an isotropic damage model with no inherent length scale [25] and the nonlocal method described in Sect. 4, comparing non-regularized and regularized results. This model postulates a Helmholtz free-energy function in coupled form

$W(\boldsymbol{C}, \zeta)=(1-\zeta) W_{0}(\boldsymbol{C})$,

where $\boldsymbol{C}=\boldsymbol{F}^{T} \boldsymbol{F}$ is the right Cauchy-Green tensor and $W_{0}(\boldsymbol{C})$ is the Helmholtz free-energy of the undamaged material. In order to accommodate large elastic deformations, we choose a compressible Neo-Hookean-type energy density $W_{0}$ that in conjunction with the singular bar solution (6.4) and the damage model (6.5) lead to large strains near the singular point $X=0$. In the particular case of one-dimensional problems the undamaged energy density may be written as

$W_{0}(\lambda)=\frac{1}{2} E\left(\lambda^{2}+\lambda^{-2}-2\right)$,

where $\lambda:=d x / d X$ is the stretch, $x=X+u$ is the position of a material point in the current configuration and $E$ is the elastic modulus. The parameter $\zeta \in[0,1]$ is the damage variable, which depends on a phenomenological variable $q$ as

$\zeta(q)=\zeta_{\infty}[1-\exp (-q / \iota)], \quad q(t)=\max _{s \in[0, t]} W_{0}(s)$,

where $\zeta_{\infty}$ is the maximum possible damage and $\iota$ is the damage saturation parameter. Using the nonlocal regularization technique of Sect. 4, the mesh dependence can be alleviated by replacing $q$ by its nonlocal counterpart

$\bar{q}=\frac{1}{\operatorname{vol}(D)} \int_{D} q d V=\frac{1}{L_{D}} \int_{L_{D}} A(X) q d X$, 


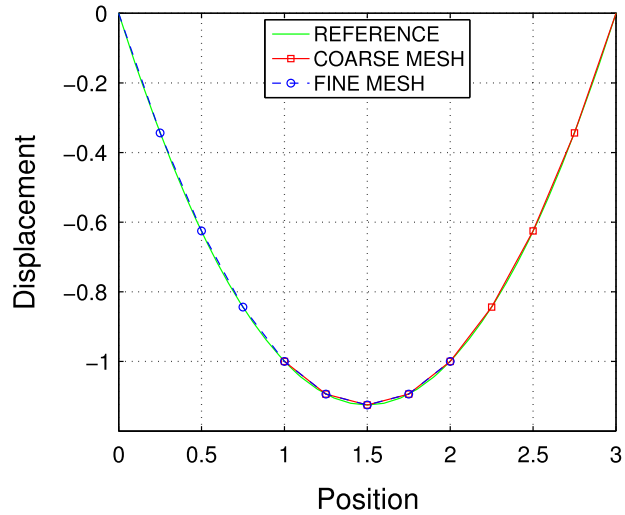

(a) Mesh Ratio $h^{\mathrm{c}} / h^{\mathrm{f}}=1, L_{2}$ coupling

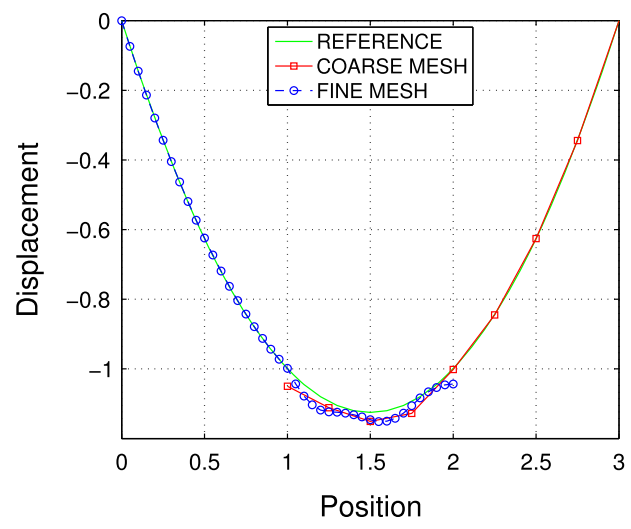

(c) Mesh Ratio $h^{\mathrm{c}} / h^{\mathrm{f}}=5, L_{2}$ coupling

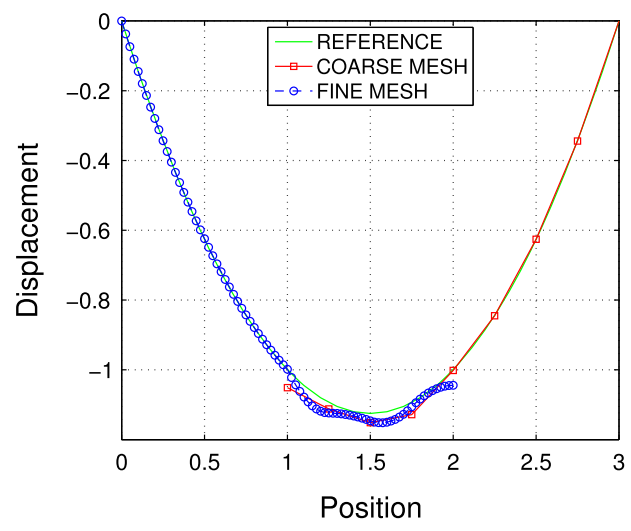

(e) Mesh Ratio $h^{\mathrm{c}} / h^{\mathrm{f}}=10, L_{2}$ coupling

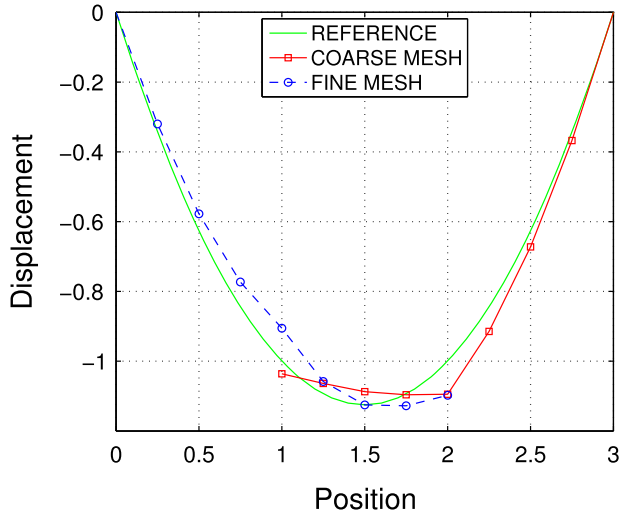

(b) Mesh Ratio $h^{\mathrm{c}} / h^{\mathrm{f}}=1, H^{1}$ coupling

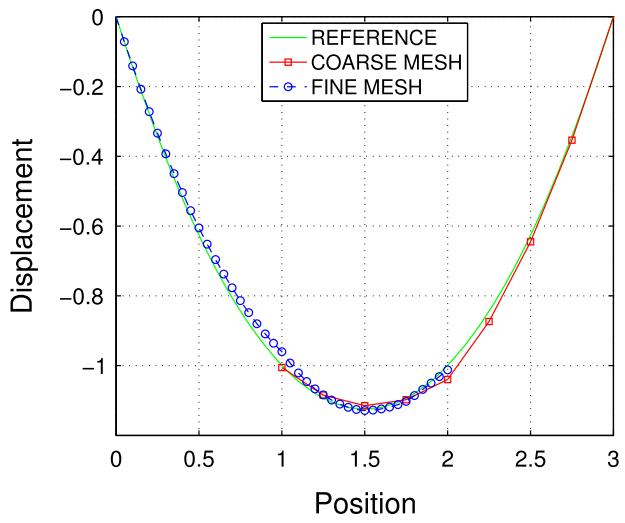

(d) Mesh Ratio $h^{\mathrm{c}} / h^{\mathrm{f}}=5, H^{1}$ coupling

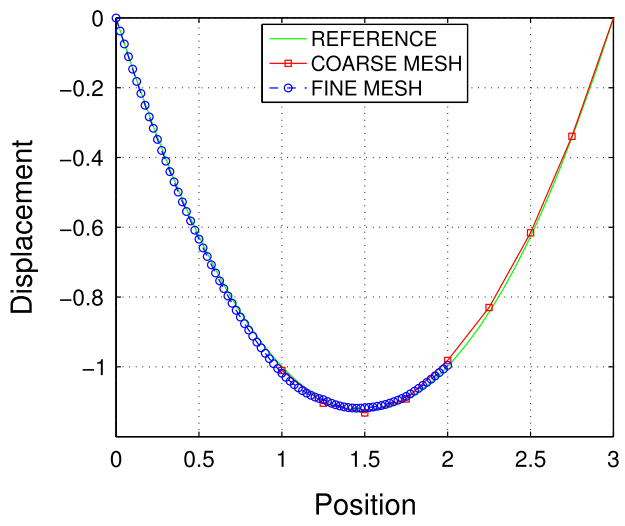

(f) Mesh Ratio $h^{\mathrm{c}} / h^{\mathrm{f}}=10, H^{1}$ coupling

Fig. 8 Clamped bar loaded by self weight. Displacement fields for constant weighing function $(\chi=0.5)$ and various mesh ratios

where $L_{D}$ is a length scale parameter.

\subsubsection{Regularized and non-regularized solutions}

The nonlocal regularization is only applied to the fine domain, while the coarse domain uses the conventional local damage model. We set the length scale parameter $L_{D}=$ $0.1 \mathrm{~m}$, with a coarse mesh size $h^{\mathrm{c}}=0.05 \mathrm{~m}$. The mesh size ratio $h^{\mathrm{c}} / h^{\mathrm{f}}$ varies from 1 to 128 . The overlapped domain is located at $X \in[0.25,0.5 \mathrm{~m}]$. The material parameters are: $E=200 \mathrm{GPa}, A_{0}=10^{-9} \mathrm{~m}^{2}, \zeta_{\infty}=1, \iota=1 \mathrm{MJ}$. We enable $H^{1}$ coupling by setting $\kappa \ell^{2}=1 \mathrm{~m}^{2}$. A $1 \mathrm{~m}$ displacement is prescribed in 100 equal-size incremental steps at $X=1 \mathrm{~m}$. Reference solutions in which both fine and coarse domains are modeled by the non-regularized damage model are also introduced for comparison. 


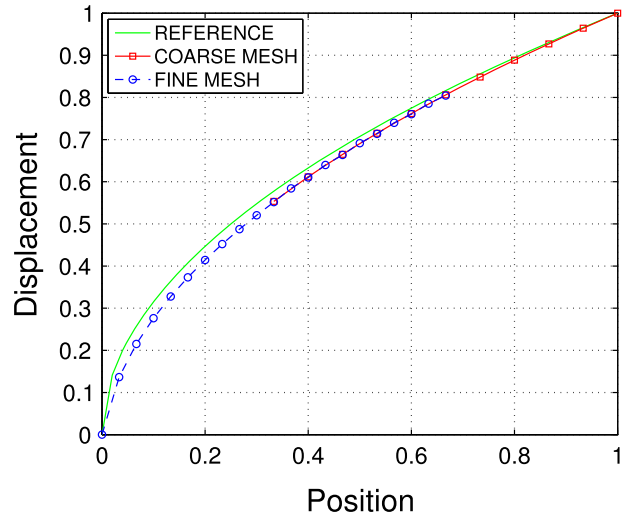

(a) Mesh Ratio $h^{\mathrm{c}} / h^{\mathrm{f}}=2$

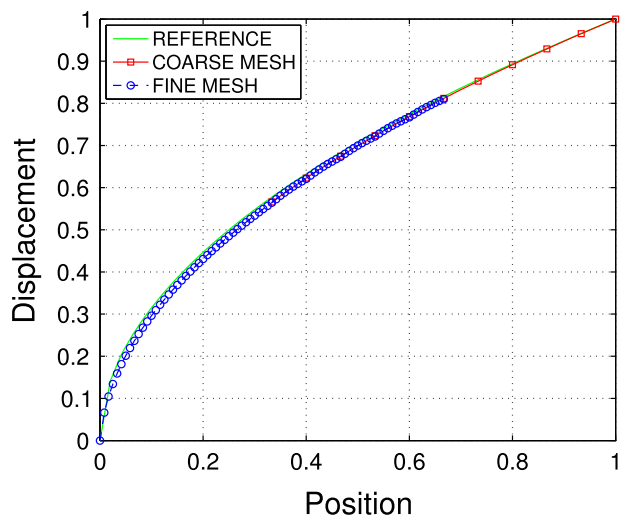

(c) Mesh Ratio $h^{\mathrm{c}} / h^{\mathrm{f}}=8$

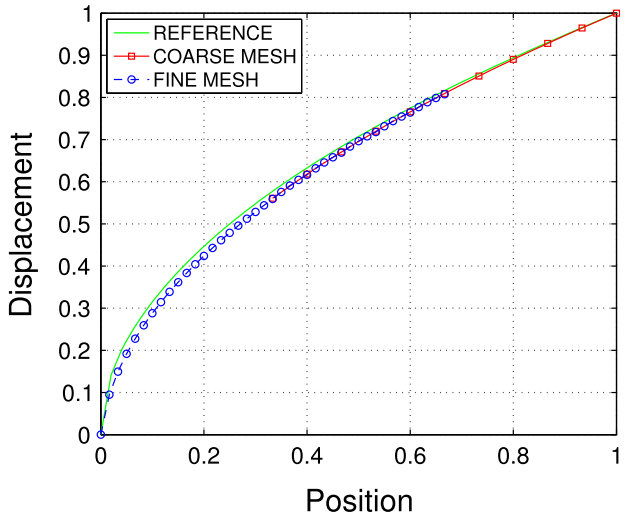

(b) Mesh Ratio $h^{\mathrm{c}} / h^{\mathrm{f}}=4$

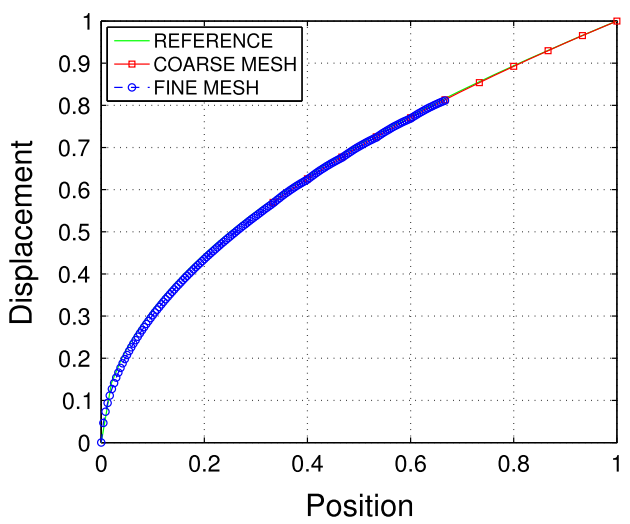

(d) Mesh Ratio $h^{\mathrm{c}} / h^{\mathrm{f}}=16$

Fig. 9 Singular bar. Solutions obtained with $H^{1}$ coupling with a constant weighing function $(\chi=0.5)$ and various mesh ratios

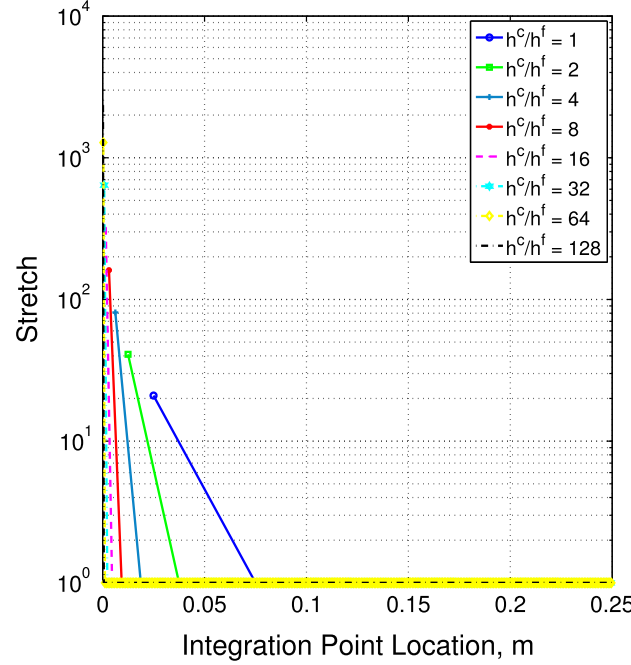

(a) Local-to-local coupling

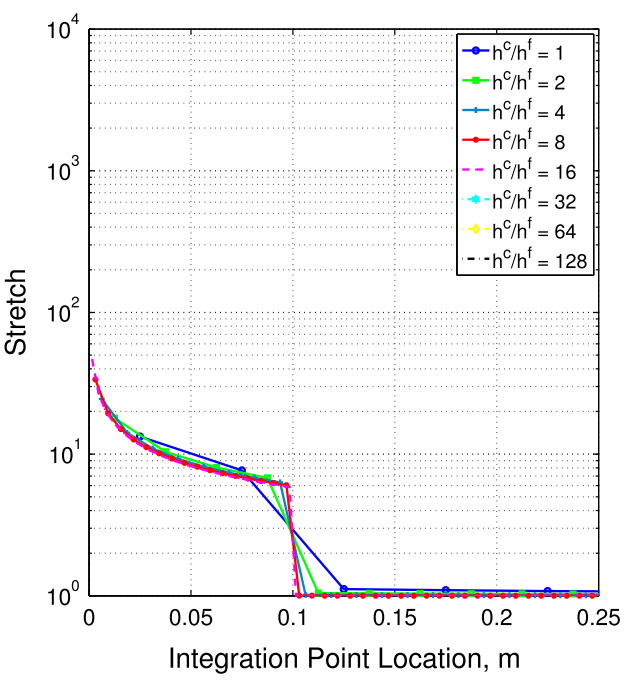

(b) Nonlocal-to-local coupling

Fig. 10 Singular bar. Stretch from $H^{1}$ coupling

Figure 10 compares the stretch field from the local-tolocal coupling and its nonlocal-to-local counterpart at the end of the simulation. For clarity, only the stretch from $X \in[0,2.5 \mathrm{~m}]$ is shown. The stretch fields obtained from the local damage model clearly exhibit mesh-dependence. Without introducing any localization limiter, the stretch localizes in the first finite element next to the singular tip. Hence, as we refine the mesh in the fine domain, we obtain stretch profiles 
Fig. 11 Singular bar. Load-displacement curves from $H^{1}$ coupling with a nonlocal damage model in the fine mesh and $\mathbf{b}$ local damage model in the fine mesh

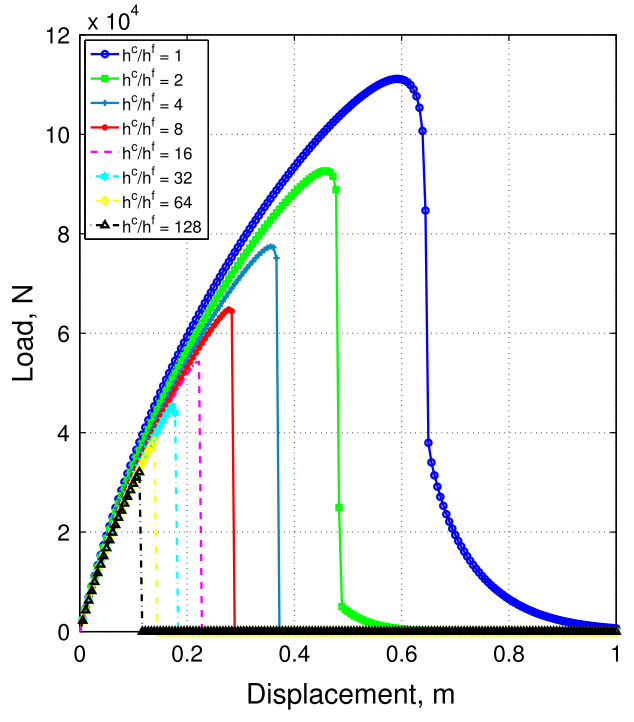

(a) Nonlocal-to-local coupling

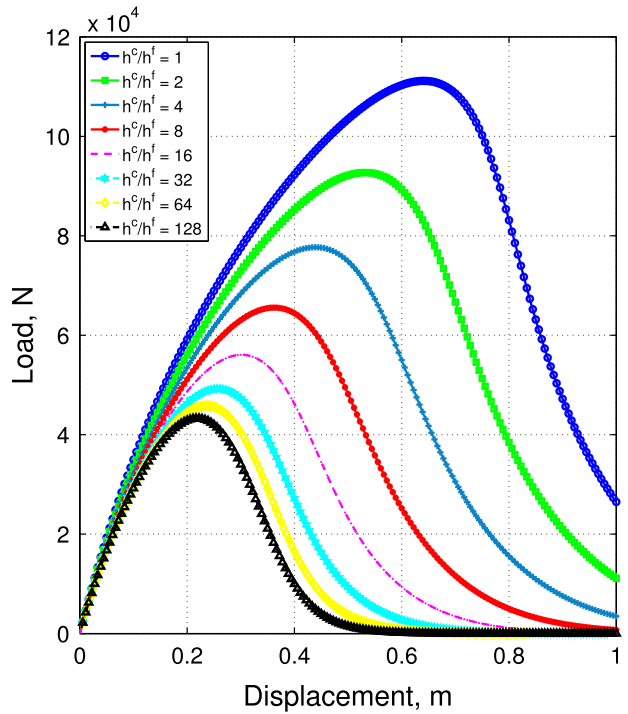

(b) Local-to-local coupling that are pathologically dependent on the fine mesh size. On the other hand, when the nonlocal damage model is used in the fine domain, we observe a stretch profile that depends on the length scale parameter $L_{D}$, but not the fine mesh size. Hence, as the mesh ratio increases, the stretch profile converges.

Figure 11 compares the load-displacement curve obtained with the fine domain modeled with and without nonlocal regularization. Due to the pathological strain localization in the first element, the load-displacement curve again exhibits mesh-dependence in the local-to-local coupling case. By contrast, due to the use of the nonlocal regularization scheme in the fine domain, the nonlocal-to-local coupling leads to a converged load-displacement curve upon refinement. Note that the nonlocal method is able to converge to a unique loaddisplacement curve (although slowly) despite the severity of the singularity in the bar.

\subsubsection{Coupling between elastic and inelastic domains}

In general, inelastic constitutive models are computationally more demanding than their elastic counterparts. As a result, significant reduction in computational costs can be obtained if the inelastic constitutive model is only confined in a region of interest where inelastic behavior is expected to take place.

In this example, we model the fine domain using the nonlocal regularization method and the coarse domain with an elastic material. Our objective here is to determine whether one can use a simple elastic model to replicate mechanical behavior in the far field, while concentrate computational resources on a small region that has a finer mesh and a more sophisticated constitutive model.

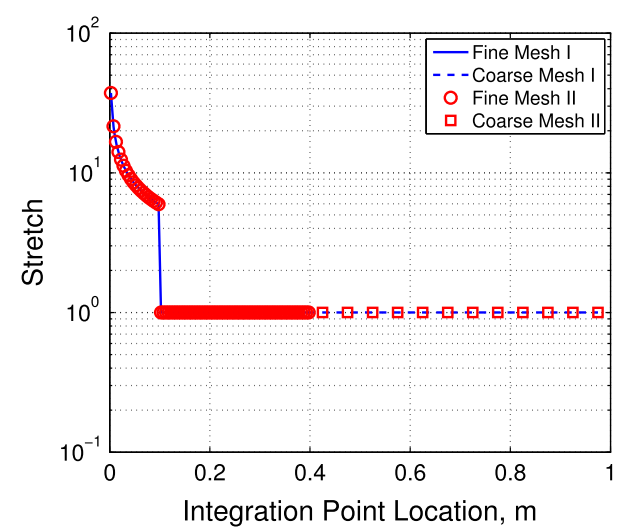

Fig. 12 Singular bar. Stretch field obtained from coupling between elastic and inelastic models (red dots) and from inelastic models only (blue line). (Color figure online)

We use again the singular bar example and set $h^{\mathrm{c}} / h^{\mathrm{f}}=10$. Figure 12 compares the two solutions, one obtained from a domain with coupling between elastic and inelastic regions (red dots) and the other with coupling between two inelastic regions that use the same inelastic model (blue line). The two yield almost identical results.

\section{Conclusion}

This work deals with energy-based domain coupling methods to enable multiscale modeling in continuum settings for strain localization purposes. To our knowledge, this is the first example of applying domain coupling methods to large deformation with inelastic materials.

Due to the introduction of the Lagrange multiplier to ensure the compatibility in the overlapped region(s), the 
resultant block system of equations may be ill-conditioned. As a result, we introduce a sequential predictor-corrector solver such that coarse and fine fields can be updated sequentially from the more well-conditioned decoupled systems. Thus, the method is also simplified by avoiding the assembly of the full system of equations.

We introduce two inf-sup tests and apply them to examine the stability of $H^{1}$ and $L_{2}$ couplings. The inf-sup tests reveal that $H^{1}$ coupling leads to much higher inf-sup values and thus exhibits more stable behavior. The introduction of the inf-sup tests provide an explanation of the spurious oscillation observed in the $L_{2}$ coupling examples in Guidault and Belytschko [23].

When strain localization occurs, it is necessary to introduce a localization limiter to avoid pathological mesh dependence. We test such a case and our results indicate that the domain coupling method is able to eliminate the mesh dependence when used in conjunction with a nonlocal regularization method.

It is desirable for computational efficiency to introduce more sophisticated constitutive models in a small domain with finer meshes and use simpler constitutive laws and coarse meshes to model the far field. This is possible to achieve with the domain coupling method provided that the fields that determine inelasticity are sufficiently small within the coupling region.

We believe for all the aforementioned reasons that the domain coupling method as presented here is a powerful tool for multiscale simulations that lead to strain localization.

Acknowledgments We thank James W. Foulk III for providing us with the analytical solution of the singular bar problem. Thanks are also due to Micheal L. Parks for suggesting the one-dimensional patch test. Support for this work was received through the U.S. Department of Energy's Advanced Simulation and Computing (ASC) Program at Sandia National Laboratories. Sandia National Laboratories is a multiprogram laboratory managed and operated by Sandia Corporation, a wholly owned subsidiary of Lockheed Martin Corporation, for the U.S. Department of Energy's National Nuclear Security Administration under contract DE-AC04-94AL85000.

\section{References}

1. Abellan Marie-Angèle, de Borst René (2006) Wave propagation and localisation in a softening two-phase medium. Comput Methods Appl Mech Eng 195(37):5011-5019

2. Aubertin P, Réthoré J, de Borst R (2009) Energy conservation of atomistic/continuum coupling. Int J Numer Methods Eng 78(11):1365-1386

3. Babuška I (1973) The finite element method with lagrangian multipliers. Numerische Mathematik 20:179-192. ISSN 0029-599X

4. Badia S, Parks M, Bochev P, Gunzburger M, Lehoucq R (2008) On atomistic-to-continuum coupling by blending. Multiscale Model Simul 7(1):381-406

5. Bathe K-J (2001) The inf-sup condition and its evaluation for mixed finite element methods. Comput Struct 79(2):243-252. ISSN 0045-7949
6. Bauman PT, Ben Dhia H, Elkhodja N, Oden J, Prudhomme S (2008) On the application of the arlequin method to the coupling of particle and continuum models. Comput Mech 42:511-530

7. Bauman PT, Oden JT, Prudhomme S (2009) Adaptive multiscale modeling of polymeric materials with arlequin coupling and goals algorithms. Comput Methods Appl Mech Eng 198(5):799 818

8. Bažant ZP, Jirásek M(2002) Nonlocal integral formulations of plasticity and damage: survey of progress. J Eng Mech 128(11):11191149

9. Belytschko T, Fish J, Engelmann BE (1988) A finite element with embedded localization zones. Comput Methods Appl Mech Eng 70(1):59-89

10. Belytschko T, Liu WK, Moran B (2000) Nonlinear finite elements for continua and structures. Wiley, New York

11. Belytschko T, Loehnert S, Song J-H (2008) Multiscale aggregating discontinuities: A method for circumventing loss of material stability. Int J Numer Methods Eng 73(6):869-894

12. Ben Dhia H (1998) Multiscale mechanical problems: the arlequin method. Compt Rendus de Acad des Sci Ser IIB 326(12):899904

13. Ben Dhia H (2008) Further insights by theoretical investigations of the multiscale arlequin metho. Int $\mathrm{J}$ Multiscale Comput Eng 6(3):215-232

14. Ben Dhia H, Rateau G (2005) The arlequin method as a flexible engineering design tool. Int J Numer Methods Eng 62(11):14421462

15. Borja RI (2000) A finite element model for strain localization analysis of strongly discontinuous fields based on standard galerkin approximation. Comput Methods Appl Mech Eng 190(1112):1529-1549

16. Borja RI (2002) Finite element simulation of strain localization with large deformation: capturing strong discontinuity using a petrov-galerkin multiscale formulation. Comput Methods Appl Mech Eng 191(27-28):2949-2978

17. Brezzi F, Marini LD (2005) The three-field formulation for elasticity problems. GAMM Mitteilungen 28:124-153

18. Brezzi F, Douglas J, Marini LD (1985) Two families of mixed finite elements for second order elliptic problems. Numerische Mathematik 47:217-235

19. Chapelle D, Bathe KJ (1993) The inf-sup test. Comput Struct 47(45):537-545

20. Chen Yi-Chao (1991) On strong ellipticity and the legendrehadamard condition. Arch Ration Mech Anal 113(2):165-175

21. Feyel F(2003) A multilevel finite element method (fe2) to describe the response of highly non-linear structures using generalized continua. Comput Methods Appl Mech Eng 192(28-30):3233-3244.

22. Fleck NA, Hutchson JW (2001) A reformulation of strain gradient plasticity. J Mech Phys Solids 49(10):2245-2271

23. Guidault PA, Belytschko T (2007) On the 12 and the h1 couplings for an overlapping domain decomposition method using lagrange multipliers. Int J Numer Methods Eng 70(3):322-350

24. Han F, Lubineau G (2012) Coupling of nonlocal and local continuum models by the Arlequin approach. Int J Numer Methods Eng 89(6):671-685

25. Holzapfel GA (2000) Nonlinear solid mechanics: a continuum approach for engineering. Wiley, West Sussex

26. Kouznetsova VG, Geers MGD, Brekelmans WAM (2004) Multiscale second-order computational homogenization of multi-phase materials: a nested finite element solution strategy. Comput Methods Appl Mech Eng 193(48-51):5525-5550

27. McAuliffe C, Waisman H (2013) Mesh insensitive formulation for initiation and growth of shear bands using mixed finite elements. Comput Mech 2:1-17 
28. Moës N, Dolbow J, Belytschko T (1999) A finite element method for crack growth without remeshing. Int J Numer Methods Eng 46:131-150

29. Mota A, Foulk JW, Ostien JT (2013) Variational nonlocal regularization in finite-deformation inelasticity. In: Proceedings of the 12th U.S. National Congress on Computational Mechanics, Raleigh, NC

30. Prudhomme S, Ben Dhia H, Bauman PT, Elkhodja N, Oden JT (2008) Computational analysis of modeling error for the coupling of particle and continuum models by the arlequin method. Comput Methods Appl Mech Eng 197(41-42):3399-3409

31. Rudnicki JW, Rice JR (1975) Conditions for the localization of deformation in pressure-sensitive dilatant materials. J Mech Phys Solids 23(6):371-394

32. Sun W, Andrade JE, Rudnicki JW (2011) Multiscale method for characterization of porous microstructures and their impact on macroscopic effective permeability. Int J Numer Methods Eng 88(12):1260-1279

33. Sun W, Chen Q, Ostien JT (2013a) Modeling hydro-mechanical responses of strip and circular footings on saturated collapsible geomaterials. Acta Geotech 5:189-198. doi:10.1007/s11440-0130276-x
34. Sun W, Ostien JT, Salinger AG (2013b) A stabilized assumed deformation gradient finite element formulation for strongly coupled poromechanical simulations at finite strain. Int J Numer Anal Methods Geomech 37(16):2755-2788

35. Toh K-C, Phoon K-K, Chan S-H (2004) Block preconditioners for symmetric indefinite linear systems. Int $\mathrm{J}$ N Methods Eng 60(8):1361-1381

36. White JA, Borja RI (2011) Block-preconditioned newton-krylov solvers for fully coupled flow and geomechanics. Comput Geosci 15:647-659.

37. White JA, Borja RI, Fredrich JT (2006) Calculating the effective permeability of sandstone with multiscale lattice boltzmann/finite element simulations. Acta Geotechn 1:195-209

38. Yang Q, Mota A, Ortiz M (2005) A class of variational strainlocalization finite elements. Int J Numer Methods Eng 62(8):10131037

39. Zhang HW, Schrefler BA, Wriggers P (2003) Interaction between different internal length scales for strain localisation analysis of single phase materials. Comput Mech 30(3):212-219 